\title{
Konfliktkommunikation für Energieinfrastrukturprojekte
}

\author{
Nils Hellmuth ${ }^{1}$ (D) $\cdot$ Eva-Maria Jakobs ${ }^{1}$
}

๑) Springer Fachmedien Wiesbaden GmbH, ein Teil von Springer Nature 2021

\section{Zusammenfassung}

Gegenstand des literaturbasierten Beitrags sind Ansätze der kommunikativen Bearbeitung von Konflikten im Kontext von Energieinfrastrukturprojekten. Das Korpus umfasst 83 Quellen. Der Fokus richtet sich auf Typen von Konflikten, Maßnahmen ihrer (kommunikativen) Bearbeitung und Zusammenhänge zwischen Konflikttyp, Konfliktgegenstand (Technologie) und Konfliktbearbeitung. Insgesamt zeigt sich deutlicher Forschungsbedarf zu passgenauen Ansätzen. Die meisten Ansätze zur (kommunikativen) Bearbeitung von Konfliktsituationen bleiben eher allgemein. Es gibt nur wenige Autoren, die die Größen Konflikttyp und Konfliktgegenstand (z.B. Windenergie versus Tiefe Geothermie) berücksichtigen. Diese betrachten primär die Typen Verteilungs-, Werte-, Wissens- und sozialer Konflikt. Die Befunde und Empfehlungen zu Konfliktkommunikation orientieren sich primär an den Beteiligungsstufen Information, Konsultation und Kooperation. Empfehlungen auf der Informationsstufe gelten als konflikttypunabhängig, Maßnahmen auf der Konsultations- und Kooperationsstufe dagegen als abhängig vom Konflikttyp. Der Größe Technologie wird weniger Einfluss zugeschrieben. Der Fokus richtet sich auf (konflikt- und technologietypbezogene) Themen und Akteursgruppen sowie Formate. Insgesamt fällt auf, dass Formate genannt und vorgestellt werden, meist jedoch konkrete Angaben zum Wie, d.h. zur konfliktmindernden kommunikativen Gestaltung der Prozesse und Formate fehlen. Hinweise zum Wie bieten vereinzelt breiter angelegte Studien zu Energiekommunikation, die ergänzend in diesem Beitrag ausgewertet wurden. Die Entwicklung von Ansätzen zur kommunikativen Bearbeitung von Konfliktsituationen, die stärker den Konflikttyp und die Technologie berücksichtigen, würden wesentlich zur Entwicklung passgenauer Gesamtstrategien für Infrastrukturprojekte der Energiewende beitragen. Dies schließt Maßnahmen zum Aufbau entsprechender Expertisen ein.

Schlüsselwörter Konfliktkommunikation · Konflikttyp · Kommunikative Konfliktbearbeitung · Konfliktanalyse · Beteiligungsstufen $\cdot$ Kommunikationsformate $\cdot$ Energieinfrastrukturprojekt $\cdot$ Energiewende

Nils Hellmuth

n.hellmuth@tk.rwth-aachen.de
Professur für Textlinguistik und Technikkommunikation, RWTH Aachen University, Aachen, Deutschland 


\title{
Conflict Communication for Energy Infrastructure Projects
}

\begin{abstract}
Subject of this literature-based contribution are approaches to the communicative processing of conflicts in the context of energy infrastructure projects. A total of 83 sources were evaluated. The focus is on types of conflicts, measures of their (communicative) processing and the connections between conflict type, conflict object (technology) and conflict management. All in all, there is a clear need for research on customized approaches. Most approaches to (communicative) processing of conflict situations remain rather general. There are only a few authors, who consider the parameters conflict type and conflict object (e.g., wind energy versus deep geothermal energy). These consider primarily the types distribution conflict, value conflict, knowledge conflict and social conflict. The findings and recommendations on conflict communication are primarily based on the participation forms of information, cooperation and consultation. Recommendations on the information level are considered to be independent of the type of conflict, whereas measures on the cooperation and consultation level depend on the type of conflict. Less influence is ascribed to the variable technology. The focus is on (conflict- and technology-type related) topics and stakeholder groups and formats. Overall, it is noteworthy that formats are mentioned and presented, but mostly lack specific information on the how, i.e., the conflict-reducing communicative design of the processes and formats. Hints on how to do this are offered by a few studies on energy communication, which were also evaluated in this article. The development of approaches to the communicative processing of conflict situations that take a greater account of the type of conflict and the technology involved would contribute significantly to the development of customized overall strategies for energy transformation infrastructure projects. This includes measures to build up corresponding expertise.
\end{abstract}

\section{Einleitung}

Die Energiewende in Deutschland ist ein sozio-technischer Transformationsprozess, der zahlreiche Infrastrukturgroßprojekte erfordert. Die Organisation und Umsetzung der Projekte ist für Politik, Planende und Durchführende herausfordernd, das damit verbundene Aufgabenspektrum komplex und vielschichtig. Die Energiewende und ihre Themen sind immanent konfliktträchtig (Renn 2015, S. 31; Krebber 2016; Bornemann und Saretzki 2018, S. 563; Brettschneider 2011, 2016a). Konfliktsituationen und Dissens sind ständige Projektbegleiter (Hoeft et al. 2017; Naumann et al. 2017); sie können (lokale) Akzeptanzprobleme verstärken. Die Sicht auf Energiethemen und Risiken divergiert zum Teil je nach Akteur stark. Die Meinungsunterschiede können zu Konfliktsituationen führen, z.B. in Bezug auf gesellschaftlich empfundene Gerechtigkeitsaspekte oder hinsichtlich eines geeigneten Standortes für Energieanlagen (Wunderlich 2012; Bauriedl 2016). Das bekannteste Konfliktbeispiel der letzten Jahre ist der Protest um den Neubau des Stuttgarter Hauptbahnhofs „Stuttgart 21“. Ein anderes Beispiel ist der Ausbau der Windenergie in Deutschland, der im Jahr 2019 mit einem Netto-Zubau von 243 Windenergieanlagen an Land und insgesamt $981 \mathrm{MW}$ nahezu zum Erliegen gekommen ist (Deutsche Windguard 2020). Der Wert liegt $80 \%$ unter dem des Rekordjahres 2017, er ist der geringste Ausbauwert seit 20 Jahren. Gründe für den schleppenden Ausbau sind lange, komplexe Genehmigungsprozesse (immissionsschutzrechtliche Genehmigung) sowie zunehmende Widerstände und Klagen von Bürgern. Die Mehrheit der Bevölkerung hat allgemein ein hohes In- teresse an Politik und Bürgerbeteiligung (z.B. Wunderlich 2012). Die Mitsprache- und Einflussmöglichkeiten in der Energiewende werden jedoch als gering eingeschätzt (Wolf et al. 2020). Insgesamt zeigt sich Unbehagen bezogen auf die Energiewendepolitik und das Lösen von Konflikten.

In der Fachliteratur wird verschiedentlich thematisiert, dass Konflikte kommunikativ bearbeitet werden müssen und Kommunikation ein wichtiger Einflussfaktor ist (Renn 2015; Becker und Naumann 2016; Brettschneider 2016a). Der vorliegende Beitrag untersucht literaturbasiert, welche Ansätze der kommunikativen Konfliktbearbeitung vorliegen, wie sich Konflikttyp und Konfliktgegenstand auf die Bearbeitung auswirken und wie Konfliktkommunikation strategisch in Kommunikationsstrategien für Technologien der Energiewende integriert werden sollte. Der Beitrag führt Befunde aus der Literatur zusammen und diskutiert diese aus kommunikationswissenschaftlicher Sicht. Ausgangspunkt ist die Konzeptualisierung von Kommunikation als Bewirkungsversuch und Angebot an den Partner (Jakobs 2019). Den übergeordneten Rahmen bildet das BMBFgeförderte Kopernikus-Projekt ENSURE II (Neue Energienetzstrukturen für die Energiewende). Ziel des Projekts ist eine zunehmend auf erneuerbare Energien ausgelegte Stromversorgung in einem Testgebiet (Energiekosmos ENSURE). Im Projekt werden neue Konzepte und Technologien des Transformationsprozesses exemplarisch umgesetzt und demonstriert mit dem Ziel einer ganzheitlichen Optimierung der Systemstrukturen. Die Umsetzung schließt die Antizipation potenzieller Konflikte (ihrer Beschaffenheit und Gründe) ein wie auch die Identifikation damit ver- 
bundener technologie-, kontext- und stakeholderbezogener Kommunikationsbedarfe.

Im Folgenden werden das methodische Vorgehen vorgestellt (Kap. 2), zentrale Begriffe wie Risiko-, Konfliktund Krisenkommunikation abgegrenzt (Kap. 3) und ausgehend von Konflikttypen (Kap. 4) Ansätze ihrer Bearbeitung (Kap. 5) beschrieben. Letzteres umfasst Mittel wie die Konfliktanalyse (Abschn. 5.1), die Zuordnung von Maßnahmen zu Beteiligungsstufen (Abschn. 5.2), Formen der konflikttypbezogenen Bearbeitung (Abschn. 5.3) und kommunikative Gestaltungsaspekte (Abschn. 5.4). Der Beitrag endet mit einem Ausblick auf Handlungsbedarf (Kap. 6).

\section{Methode}

Die Studie nutzt ein mehrstufiges, iteratives Design. Ausgangspunkt des Beitrags waren die Studien von Ziekow et al. (2014b, c, d, e, f) und das Interesse, literaturbasiert das Zusammenspiel von Technologie, Konflikttyp und Formen seiner kommunikativen Bearbeitung vertiefend zu betrachten. Konflikte werden in diesem Beitrag primär in ihrer Entstehung betrachtet, nicht jedoch als Teil von Krisen. Die Fokussetzung auf Deutschland ergibt sich maßgeblich aus der Zielsetzung von ENSURE II; sie stützt sich zugleich auf Befunde der Technikakzeptanz- sowie der Sprach- und Kommunikationsforschung. Erstere zeigen, dass die Wahrnehmung konkreter Energiemaßnahmen häufig stark durch lokale Standortfaktoren beeinflusst wird (vgl. etwa Wunderlich 2012). Letztere verstehen Kommunikation als Bewirkungsversuch und stark durch Kulturen und Sprachräume überformtes Phänomen (Jakobs 2019). Aus den genannten Gründen beschränkt sich die Studie weitgehend auf deutschsprachige Publikationen zum Thema.

Die Auswertung der Fachliteratur erfolgte in drei Schritten. Die Recherche folgt methodisch van Wee und Banister (2015) sowie Rowley und Slack (2004). Die qualitative Auswertung orientiert sich an Mayring (2010).

Schritt 1 dient der Klärung zentraler Begriffe (Konflikt, Konflikttyp, Konfliktkommunikationen) und ihrer Abgrenzung gegen verwandte Begriffe (Risiko und Krise). Die Recherche erfolgte schlagwortbasiert mit den Keyword-Kombinationen (Verknüpfung mit dem Booleschen Operator AND) Konflikt/Risiko, Konflikt/Krise, Konflikt/Risiko/Krise, Konfliktkommunikation/Risikokommunikation/KrisenKommunikation sowie dem Schlagwort Konflikttyp. Wo möglich, wurden Filter verwendet: Deutsch, Sozialwissenschaften/ Humanities, zusätzliche Wortformen. Die Suche erfolgte mit drei Instrumenten (Rowley und Slack 2004): in der Datenbank der Hochschulbibliothek der RWTH Aachen University (für einen ersten Überblick), mit Suchmaschinen (Google Scholar, Microsoft Academic, Bielefeld Academic Search Engine (BASE)) sowie in Online-Literaturdatenban- ken (Web of Science, National Library of Energy, Scopus). Die Abfrage in der Hochschulbibliothek ergab 73 Treffer. Die Abfrage per Suchmaschinen brachte die meisten Treffer. Die Abfrage in den Online-Literaturdatenbanken brachte kein Ergebnis (mit Ausnahme von Scopus: 1 Treffer); die Datenbanken indexieren primär englischsprachige Forschung.

Die Rechercheergebnisse per Suchmaschine variieren stark abhängig von der Suchmaschine und wurden teilweise durch weitere Filter reduziert. Die Suche in Bielefeld Academic Search Engine ergab 35 Treffer, die Suche in Google Scholar und Microsoft Academic dagegen sehr hohe Trefferzahlen (Google Scholar: 492.127 Treffer; Microsoft Academic: 10.569 Treffer). In einem zweiten Durchlauf wurden alle Befunde gesichtet (Titel, Abstract) und stark reduziert (Löschen von Doppelnennungen, thematisch nicht relevanten Beiträgen; suchmaschinentyp-bedingte Fehler, in Google Scholar kann z.B. das Kriterium Sozialwissenschaften nicht als Filter eingegeben werden). Es wurden nur Beiträge berücksichtigt, die sich im Kern mit Konflikttypen befassen und/oder die als Überblicksdarstellung Unterschiede von Konflikt/Risiko/Krise oder Konfliktkommunikation/Risikokommunikation/Krisenkommunikation diskutieren. Das Korpus $(n=28)$ wurde bezogen auf folgende Fragen ausgewertet: Wie sind die Begriffe Risiko, Konflikt und Krise definiert und was unterscheidet sie? Wie werden Risiko-, Konflikt- und Krisenkommunikation voneinander abgegrenzt? Welche Konflikttypen werden in der Literatur unterschieden? Dazu wurden Definitionen extrahiert und bezogen auf Gemeinsamkeiten und Unterschiede verglichen.

Schritt 2 fokussiert Publikationen, die sich mit dem Zusammenhang von Konflikt(-typ), Technologie und kommunikativer Konfliktbearbeitung bei Energieinfrastrukturprojekten befassen. Die Suche erfolgte schlagwortbasiert mit den Keyword-Kombinationen Konflikt/Technologie, Konflikt/Infrastrukturprojekt, Konfliktbearbeitung/Infrastrukturprojekt und Konfliktkommunikation/Infrastrukturprojekt. Wie in Schritt 1 wurden - wenn möglich - die Filter Deutsch, Sozialwissenschaften/Humanities, zusätzliche Wortformen sowie der Boolesche Operator AND verwendet. Die Suche erfolgte im Datenbestand der Hochschulbibliothek der RWTH Aachen University und mit Suchmaschinen (Google Scholar, Microsoft Academic, Bielefeld Academic Search Engine). Der Abruf über die Hochschulbibliothek ergab vier Treffer. Die Recherche in der Bielefeld Academic Search Engine ergab 10 Treffer. Deutlich höher war die Trefferanzahl bei Google Scholar (99.035 Treffer) und Microsoft Academic (8120 Treffer). Die Treffer wurden gesichtet (Titel, Abstract) und reduziert auf relevante Funde (Löschen von Doppelnennungen, thematisch nicht relevanten und fachfremden Beiträgen (Signalwörter z. B. Medizin oder Pädagogik); 


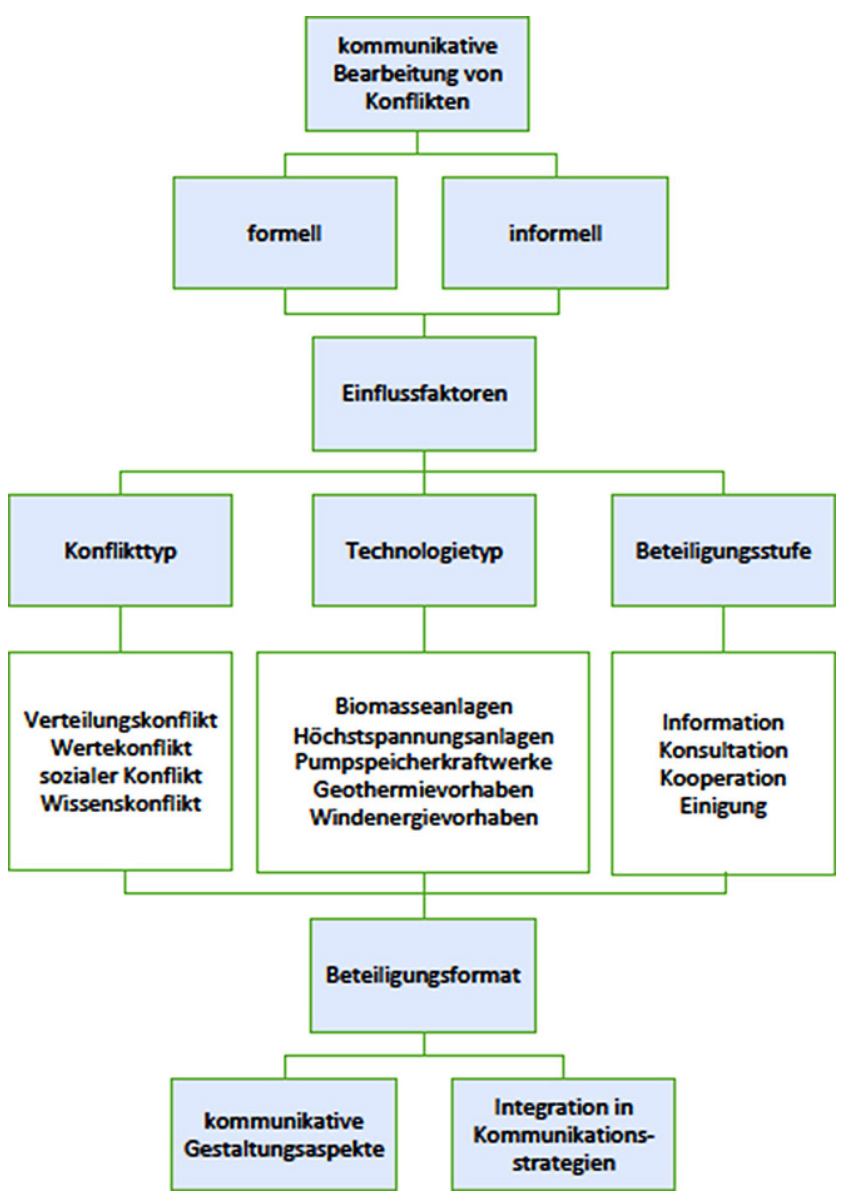

Abb. 1 Ausschnitt des Kategoriensystems

suchmaschinentyp-bedingte Fehler). Es wurden nur Beiträge berücksichtigt, die sich auf Konflikte im Kontext von Infrastrukturprojekten im Energiesektor beziehen (Auswahlkriterium). Aussortiert wurden u. a. Texte zu (welt)politischen Konflikten (z. B. zwischen Staaten) oder Konflikten in bzw. zwischen Unternehmen und Organisationen. Zur Überprüfung der Reliabilität der Recherche wurde die Suche parallel von verschiedenen Personen durchgeführt und die Ergebnisse abgeglichen. Das Korpus 2 wurde später bei der Auswertung der Publikationen nach dem Schneeballprinzip (Hinweis auf themenrelevante Quellen) ergänzt. Dies führte u.a. zur Anreicherung des Korpus mit ausgewählten englischsprachigen Publikationen. Das finale zweite Korpus enthält 22 neue Texte. Das Korpus wurde inhaltsanalytisch (top-down, bottom-up) ausgewertet bezogen auf folgende Fragen: Welche Ansätze gibt es für die (kommunikative) Bearbeitung von Konflikten? Welche Mittel werden für welchen Konflikttyp empfohlen? Wird ein Zusammenhang zwischen Konflikttyp, kommunikativer Bearbeitung und Technologietyp gesehen? Wenn ja, welcher Art ist der Zusammenhang? Die Kategorien (Oberund Unterkategorien) wurden fragegeleitet und induktiv aus dem Material abgeleitet, definiert und während der
Analyse überprüft und ggf. überarbeitet (Mayring 2010, S. 59), um Nachvollziehbarkeit und Intersubjektivität des Vorgehens zu sichern. Abb. 1 zeigt einen Ausschnitt des entwickelten Kategoriensystems.

Schritt 3: Schritt zwei ergab, dass nur wenige Autoren und dann vereinzelt auf die konkrete Gestaltung von Konfliktkommunikation, z.B. Einsatz und Gestaltung von Formaten, und damit verbundene Herausforderungen eingehen. Dies führte zu dem Entschluss, den Blick auf Literatur zur kommunikativen Begleitung von Energieinfrastrukturprojekten $\mathrm{zu}$ erweitern. In einer ergänzenden Recherche wurde weitere Literatur anhand der KeywordKombinationen Kommunikation/Infrastrukturprojekt, Kommunikationsstrategie/Infrastrukturprojekt und Guideline/ Report/Leitfaden/Ratgeber Infrastrukturprojekt identifiziert (Hochschulbibliothek RWTH Aachen: 0 Treffer, BASE: 35, Google Scholar: 10.289, Microsoft Academic: 30.470). In einem zweiten Durchlauf wurde das Suchergebnis auf Publikationen verdichtet, die sich im Kern mit dem Thema befassen und im Laufe der Analyse das Korpus nach dem Schneeball-Prinzip ergänzt. Das Korpus $3(n=33)$ wurde analog zu Schritt 2 inhaltsanalytisch ausgewertet. Den Ausgangspunkt bildeten folgende Fragen: Welche Empfehlungen werden für die kommunikative Begleitung von Infrastrukturprojekten gegeben (Formate, Qualitätskriterien, sprachlich-visuelle Gestaltung)? Wo zeigen sich Bezüge zur Konfliktkommunikation?

\section{Risiko - Konflikt - Krise}

Konflikte zeigen enge Bezüge zu Risiken und Krisen. Risiken können zu Konflikten führen, Konflikte können Krisen nach sich ziehen. Im Folgenden werden die Begriffe Risiko, Konflikt und Krise sowie Risiko-, Konflikt- und Krisenkommunikation in ihrem potenziellen zeitlich-kausalen Zusammenhang betrachtet und für die Zwecke des Beitrags voneinander abgegrenzt.

Die Ursprünge des Begriffs Risiko sind alt. Neben ersten Verwendungen in Mesopotamien und China lassen sich dem Begriff arabische, altitalienische oder griechische Wurzeln zuordnen (Ruhrmann 2015). Im Griechischen bezeichnet Risiko das Umschiffen einer Klippe und die damit verbundene Gefahr. Weitze und Renn (2019, S. 11) fassen Risiko als die Möglichkeit des Eintreffens einer Handlung oder eines Ereignisses und die direkt damit zusammenhängenden erwarteten Konsequenzen, die abhängig von der subjektiven Bewertung der Handlung bzw. des Ereignisses positiv oder negativ ausfallen können. Negativ bewertete Folgen werden als Risiko, eher positiv bewertete Folgen als Chance bezeichnet (Wiedemann et al. 2000).

Wenn sich die Handlung bzw. das Ereignis an einem sensiblen Punkt negativ entwickelt, kann daraus ein Kon- 
flikt erwachsen. Unter einem Konflikt wird eine Auseinandersetzung von mindestens zwei Parteien zu einem Gegenstand oder zu einem Thema verstanden (Kepplinger 2015). Dabei kann es sich um einen privaten (z.B. in der Familie oder zwischen Freunden), öffentlichen (z. B. Mieterstreit vor anderen Hausbewohnern) oder publizistischen Konflikt (in den Massenmedien) handeln (ebd.). Nach Dahrendorf (1961) sind Konflikte ein ,notwendiges Element allen gesellschaftlichen Lebens“ und umfassen ,alle strukturell erzeugten Gegensatzbeziehungen“. Beim Konflikt lehnt ein beteiligtes System das selektive Kommunikationsangebot oder den Selektionsvorschlag eines anderen beteiligten Systems ab und macht dies zum Thema weiterer Kommunikation (Krause 1996, zitiert nach Schulz 2001, S. 21). Die Bundesregierung (2019) sieht Konflikte als „natürlicher Bestandteil gesellschaftlicher Veränderungsprozesse“; ein „Konflikt gehört zur Normalität von Demokratie“ (RWE 2012) und ist Teil des menschlichen Bewusstseins in verschiedenen Aspekten des Lebens. Konflikte treten häufig bei kontrovers diskutierten Technologien auf (z.B. Gentechnik, künstliche Intelligenz oder Hydraulic Fracturing) wie auch infolge wahrgenommener Risiken von Infrastrukturprojekten (z. B. beim Stromnetzausbau der Einfluss elektromagnetischer Felder oder Folgen der Projektumsetzung wie Geruchsbelästigung oder Lärm). Konflikte können Reaktionen auf Risiken oder der Ausgangspunkt für Krisen sein.

Eine Krise kann nach Drews (2018) auf individueller (persönliche Probleme, z.B. Unfall oder Krankheit), organisationaler und/oder gesellschaftlicher Ebene stattfinden. Heath und O'Hair (2009) konzeptualisieren eine Krise als ein manifestiertes Risiko. Der Begriff stammt vom griechischen krisis bzw. vom lateinischen crisis und bedeutet soviel wie Wendepunkt (Fröhlich et al. 2015). Eine Krise entsteht, wenn das Eintreffen eines Risikoereignisses nicht verhindert werden konnte und sich der Konflikt zuspitzt und schließlich eskaliert, weil er nicht behoben werden konnte (Auslöser der Krise). Eine Krise ist nicht intendiert, meist unvorhergesehen und negativ und stellt ,eine ernsthafte Bedrohung für grundlegende Strukturen, Werte oder Normen eines gesellschaftlichen Systems sowie für das System selbst dar und damit für lebensnotwendige Infrastrukturen in einer Gesellschaft" (Drews 2018, S. 51). Ein bekanntes Beispiel ist die Reaktorkatastrophe im japanischen Fukushima im Jahr 2011, die Krisen auf allen drei oben genannten Ebenen auslöste. Die Risiken der Atomenergienutzung waren vor und nach dem GAU gleich hoch, wurden jedoch unterschiedlich wahrgenommen. Was sich nach dem GAU änderte, war die Wahrnehmung großtechnischer Risiken durch die breite Bevölkerung. Die Risiken wurden nicht mehr abstrakt wahrgenommen (der GAU im sowjetischen Atomkraftwerk von Tschernobyl lag bereits 25 Jahre zurück), sondern als Risiken mit ganz realen Aus- wirkungen (Renn 2011). In Deutschland führte das Ereignis zu der Rücknahme von Laufzeitverlängerungen für Kernkraftwerke und zu dem Beschluss, Ende des Jahres 2022 aus der Kernenergie auszusteigen. Wenn Krisensituationen eintreten, muss unmittelbar gehandelt und die Öffentlichkeit darüber informiert werden (Borg et al. 2018) - dies gilt in abgeschwächter Form auch für den Konflikt.

In der Literatur werden die Bereiche Risiko- und Krisenkommunikation häufiger betrachtet als der Bereich Konfliktkommunikation. Die Literatur zu Konfliktkommunikation thematisiert eher selten Konflikte bei Infrastrukturprojekten im Energiesektor. Die meisten Studien befassen sich mit Konfliktkommunikation zu anderen Anwendungsgebieten. Für den Energiesektor gibt es jedoch einige Einzelanalysen (mit diversen Schwerpunkten), Sammelbände (u. a. Feindt und Saretzki 2010; Holstenkamp und Radtke 2018) oder Monographien (z. B. Schmalz 2019).

Risikokommunikation umfasst den Austausch von Informationen und Meinungen zu Risiken durch Individuen oder Gruppen (Weitze und Renn 2019, S. 13) und „beschreibt den Prozess, der die Unsicherheit des Eintritts eines Schadens und die Ungewissheit zukünftiger Schadensfolgen problematisiert" (Ruhrmann 2015). Dabei geht es um die Art, das Ausmaß, die Bedeutung oder die Kontrolle eines Risikos (Covello 1992). In der Literatur wird Risikokommunikation mit der Identifikation von Risiken in Verbindung gebracht und/oder mit der Produktion öffentlicher Darstellungen (Reynolds und Seeger 2005). Die Darstellungen beziehen sich auf Gesundheits- und Umweltrisiken (wie auch technische, ökonomische, infrastrukturelle oder die Privatsphäre betreffende Risiken) oder auf die Frage, wie Negativfolgen reduziert oder vermieden werden können (ebd.), z.B. bei Technologien der Geothermie, die von einem Großteil der Bevölkerung als Risikotechnologien wahrgenommen werden (Weber und Brian 2014). Die Bereiche Umwelt und Gesundheit dominieren thematisch (vgl. Drews 2018). Im Bereich der Gesundheitskommunikation wird Risikokommunikation häufig mit dem Bestreben verbunden, die Öffentlichkeit von weniger riskanten Verhaltensweisen $\mathrm{zu}$ überzeugen und sie zu einer gesünderen Lebensweise zu motivieren (Freimuth et al. 2010, zitiert nach Reynolds und Seeger 2005). Als Funktionen der Risikokommunikation gelten laut Renn (2015) die Aufklärung über wissenschaftliche Forschung zu positiv und negativ zu erwartenden (Neben-)Wirkungen, die Koordination betroffener Akteure bezüglich möglicher Schutzmaßnahmen und Verhaltensanpassungen, die umfassende Information über eingesetzte Verfahren zur Bewertung und Abwägung von Risiken, die Klärung unterschiedlicher Standpunkte sowie die Durchführung kommunikativer Beteiligungsverfahren. Als Formen der Zielumsetzung werden genannt: Dokumentation, Information, Dialog und Beteiligung (ebd.). Risikokommunikation soll kontrolliert, regelmäßig und strukturiert ab- 
laufen sowie langfristig angelegt sein (Reynolds und Seeger 2005, S. 48f.).

Krisenkommunikation hat sich wie Risikokommunikation zu einem eigenständigen Forschungsfeld entwickelt (Drews 2018). Krisenkommunikation bezeichnet die Art und Weise der Kommunikation während einer Krisensituation (Kepplinger 2015) und steht für den „Aushandlungsprozess im Kontext von als bedrohlich und disruptiv wahrgenommenen Situationen, denen Beobachter intuitiv oder strategisch den Krisenstatus zuschreiben" (Schwarz 2015). Krisenkommunikation wird typischerweise mit der Öffentlichkeitsarbeit von Unternehmen und Organisationen in Verbindung gebracht; der Begriff subsumiert Bemühungen, die öffentliche Wahrnehmung eines Ereignisses durch strategisch ausgerichtete Kommunikationsprozesse zu steuern und so zu gestalten, dass der Schaden sowohl für die Organisation als auch für die Interessensgruppen verringert wird (Reynolds und Seeger 2005).

Konfliktkommunikation wird in der Literatur mehrheitlich als Mittel gesehen, mögliche Konfliktfelder (und das damit verbundene Konfliktpotential) präventiv zu identifizieren (u.a. Kepplinger 2015) oder bereits bestehenden Konflikten - insbesondere in Beteiligungsverfahren - entgegenzuwirken. Konflikte sollen konstruktiv aufgearbeitet werden und im respektvollen, gegenseitigen Aufeinanderzugehen bearbeitet und gelöst werden - vor diesem Hintergrund kann ein Konflikt auch als Chance gesehen werden. Eher wenige Autoren gehen auf das Wie der Gestaltung und auf die Umsetzung von Konfliktkommunikation ein, z.B. wie Formate gestaltet werden sollten oder welchen Qualitätskriterien sie genügen müssen, um effizient zur Konfliktbearbeitung beitragen zu können. Einige wenige diskutieren dies bezogen auf den vorliegenden Konflikttyp.

\section{Konflikttypen}

\subsection{Einteilungsversuche}

In der Literatur werden verschiedene Typen von Konflikten genannt. Die Einteilungen sind divers; sie differieren je nach Autor, Ansatz und Fachrichtung. Als wichtige, häufig auftretende Konfliktgründe und -auslöser gelten der Verteilungs- bzw. Interessenskonflikt, der Wissenskonflikt und mit leichten Abstrichen der Wertekonflikt (Aubert 1963; Conrad 2010; Geis 2010; Ohlhorst und Schön 2010; Duve et al. 2011; Ziekow et al. 2014a). Einige Autoren (z.B. Benighaus et al. 2010; Bogner und Menz 2010; Hampel und Torgersen 2010) unterscheiden zwischen Wissenskonflikt (teilweise als kognitiver Konflikt bezeichnet) und Nichtwissenskonflikt (vgl. Feindt und Saretzki 2010), und betrachten diese als relevant. Andere Autoren sehen die Annahme von (Nicht-)Wissenskonflikten als eigene Kate- gorie(n) kritisch und nennen stattdessen Interessens- und Wertekonflikt als elementare Konflikttypen (Feindt und Saretzki 2010, S. 14, 22; z. B. Böschen 2010; Fischer 2010). Die Kategorie Wertekonflikt wurde vom Soziologen Aubert (1963, zitiert nach Böschen 2010, S. 106) als gegensätzliche Position zum Interessenskonflikt eingeführt, weil hier nicht der Streit um die Verteilung knapper Güter im Vordergrund steht, sondern der Streit um deren Bewertung (Wertekonflikte betreffen u.a. die Stammzellenforschung oder Präimplantationsdiagnostik). Der Interessenskonflikt (nach Aubert 1963) zeigt viele Überschneidungen mit dem Verteilungskonflikt, weil die Berücksichtigung der eigenen Interessen mit einer gerechten Verteilung korreliert (Geis 2010; C.A.R.M.E.N. 2014; Ziekow et al. 2014a; Becker und Naumann 2016). Beim Verteilungskonflikt verstärken die Auseinandersetzungen um eine (gerechte) Verteilung knapper Güter und das Durchsetzen gegenseitiger Interessen oft bereits bestehende soziale Konflikte (bei Ziekow et al. 2014b der vierte Konflikttyp). Geis (2010) nennt neben Verteilungs-, Wert- und Wissenskonflikten als eigene Kategorie den Machtkonflikt. Conrad (2010) differenziert zwischen konsensualen und dissensualen Konflikten, aus denen Interessens-, Werte- und Mittelkonflikte entstehen können. Die Juristen Duve et al. (2011) nennen Beziehungs-, Strategie- und Sachkonflikt; C.A.R.M.E.N. (2014) nennen diese und den Verteilungskonflikt sowie ergänzen den Wertekonflikt um den Grundsatzkonflikt. Ohlhorst und Schön (2010) unterscheiden - speziell bei Windenergieprojekten - Technik- und Strategiekonflikt, Interessens- und Machtkonflikt sowie Werte- und Zielkonflikt. Weitere im Zusammenhang mit erneuerbaren Energien genannte Konflikttypen sind Verfahrenskonflikt, Standort- bzw. Landnutzungskonflikt, Identitätskonflikt, Energieträger- bzw. technologischer Konflikt (Becker und Naumann 2016 Naumann et al. 2017) sowie Knappheits-, Mensch-Natur- und Ressourcennutzungskonflikt (Ackermann et al. 2001; Scheffran 2010).

Je nach Betrachtungsweise (einzelner Gruppen) können für denselben Konfliktaspekt gleichzeitig mehrere Konflikttypen vorliegen, z.B. Verteilungskonflikt von Ressourcen und Wertekonflikt bei der Priorisierung von Eigenschaften (Becker und Naumann 2016). Es kann auch ein Wertekonflikt vorliegen, dieser aber einen anderen Konflikt (z. B. bestehenden Verteilungskonflikt) maskieren (Ziekow et al. 2014b) - die Auswirkung (Intensität) eines Konflikttyps auf eine Technologie kann variieren. Ein Verteilungskonflikt kann sich von der Sachebene auf die Beziehungsebene verlagern und dann gleichzeitig (auch) ein Beziehungskonflikt sein. Technik- und Strategiekonflikte können sich auf das Kosten-Nutzen-Verhältnis eines Vorhabens wie auch auf eine gerechte Verteilung beziehen; infolgedessen können mehrere Konflikttypen gleichzeitig auftreten und sich gegenseitig beeinflussen (z. B. ein Technik- und Strategiekonflikt einen Verteilungskonflikt). 
Ziekow et al. (2014b, c, d, e, f) betrachten Konfliktkommunikation bezogen auf die Bearbeitung möglicher Konflikte in Planungs- und Genehmigungsverfahren. Ihre Empfehlungen und Ausführungen adressieren primär Behörden. Die Autoren unterscheiden vier Konflikttypen Verteilungs-, Werte-, Wissenskonflikt und sozialer Konflikt -, die bei großen Infrastrukturprojekten kombiniert auftreten können (Ewen et al. 2013). Ziekow et al. betrachten die vier genannten Konflikttypen bezogen auf fünf Technologietypen: Biomasseanlagen (Bioenergie) (Ziekow et al. 2014b), Höchstspannungsleitungen (Ziekow et al. 2014c), Pumpspeicherkraftwerke (Wasserkraft) (Ziekow et al. 2014d), Tiefen-Geothermievorhaben (Erdwärme) (Ziekow et al. 2014e) und Windenergieanlagen (Ziekow et al. 2014f). Explorative Untersuchungen von Becker et al. (2012) in Brandenburg deuten darauf hin, dass keine Energieform vor Konflikten durch Bürgerproteste geschützt ist. Die Technologietypen lassen sich nach Brettschneider (2016a, S. 220) der Kategorie „Energieinfrastrukturprojekt“ zuordnen. Im Folgenden werden die von Ziekow et al. betrachteten Konflikttypen (Abschn. 4.2-4.5) in ihrem Bezug zu Technologietypen vorgestellt.

\subsection{Verteilungskonflikt}

Bei Verteilungskonflikten geht es um die Verteilung positiv oder negativ bewerteter Objekte, die nur in begrenzter Menge zur Verfügung stehen. Zumeist entsteht der Konflikt durch eine Ungleichverteilung direkter Vor- und Nachteile, wobei zwischen ökonomisch-materiellen Vor- und Nachteilen und immateriellen Nachteilen unterschieden wird (Ziekow et al. 2014b). Auf immaterielle Vorteile (z. B. angrenzende Flächen dürfen aufgrund des Infrastrukturausbaus nicht als Baugrund für Wohneigentum genutzt werden) wird nicht näher eingegangen. Die Konfliktparteien versuchen, möglichst viele Vorteile für sich selbst zu erzielen. Bei jedem Vorhaben profitiert mindestens eine Gruppe vom Infrastrukturausbau (Nutzen), während andere materielle bzw. immaterielle Nachteile hinnehmen müssen (Kosten). Eine von allen Parteien als gerecht empfundene Verteilung stellt eine große Herausforderung dar. Die Themen und Gründe, die $\mathrm{zu}$ Verteilungskonflikten führen, differieren meist abhängig vom Technologietyp - es gibt aber auch Gemeinsamkeiten. Ein ökonomischer Nachteil, der für jeden Technologietyp gleich ist, ist die Sorge von Hauseigentümern, die eigene Immobilie könne an Wert verlieren (Ziekow et al. 2014b, c, d, e, f). Ein anderer ökonomischer Vor- bzw. Nachteil betrifft die Gruppen, die durch den Infrastrukturausbau Geld verdienen oder mit Belastungen konfrontiert werden; beim Bau von Windenergieanlagen erwirtschaften z.B. Landwirte Geld, wenn sich ihr Land als Ausbaufläche eignet, während benachbarte Landwirte oder benachbarte Anwohner leer ausgehen - beide Parteien müssen aber mit den Nachteilen des neuen Windrads leben (Ziekow et al. 2014f). Immaterielle Nachteile aller Technologietypen betreffen eine potenzielle gesundheitliche Beeinträchtigung, die Gründe differieren technologiespezifisch. Bei Höchstspannungsleitungen geht es um $\mathrm{zu}$ hohe Immissionen durch elektrische und magnetische Felder, bei Tiefen-Geothermie um Schadstofffreisetzung radioaktiver Elemente oder Grundwasserverschmutzung, bei Windenergie um die Auswirkungen von Infraschall (Ziekow et al. 2014c, e, f). Weitere immaterielle Nachteile betreffen den Verlust von Lebensqualität der Anwohner durch Lärm und Geräuschentwicklung (u.a. durch erhöhten LKW-Verkehr oder Schall) sowie Veränderungen des Landschaftsbilds, etwa durch Vermaisung (auch: Eltrop et al. 2014). Windenergieanlagen können zur Belästigung durch Schlagschatten führen, Biomasseanlagen $\mathrm{zu}$ Geruchsbelästigungen. Beim Bau von Höchstspannungsleitungen treffen potenzielle negative Auswirkungen die umgebende Natur (Vogelarten), bei Tiefen-Geothermie seismische Ereignisse (Erderschütterungen, Hebungen oder Senkungen); bei Pumpspeicherkraftwerken kann das Grundwasser versiegen (Ziekow et al. 2014b, c, d, e, f).

\subsection{Wertekonflikt}

Der zweite Konflikttyp gründet auf unterschiedlichen Wertvorstellungen und der Frage, ob ein Energieinfrastrukturprojekt ethisch oder normativ sinnvoll ist (Ziekow et al. 2014b). Werte sind subjektiv; sie gelten als positiv und als erstrebenswerte Eigenschaften und Ideale. Sie werden dem Menschen durch unterschiedliche Einflüsse vermittelt, z. B. durch das Elternhaus, die Gesellschaft oder die Religionszugehörigkeit. Menschen handeln wertebasiert, Werte können sich aber im Laufe eines Lebens auch ändern, weswegen es - begrenzte - Möglichkeiten der Reaktion auf wertebasierte Kritik gibt. Die Möglichkeiten der Konfliktminderung hängen vom Vorhaben $a b$. Ein unabhängig vom Technologietyp omnipräsenter Wert ist der Klimaschutz, in Konkurrenz dazu stehen der Natur-, Landschafts- und Artenschutz sowie die Erhaltung der Biodiversität (Ziekow et al. 2014b, c, d, e, f; innerökologische Konflikte bei Brettschneider 2016b). Weitere Wertekonflikte betreffen divergierende Ansichten zum Vorrang wirtschaftlicher Interessen (Ökonomie), zum Naturschutz (Ökologie) (z. B. bei Pumpspeicherkraftwerken) oder zur Bewertung von Risiken (z. B. bei Tiefen-Geothermie) (Ziekow et al. 2014b, c, d, e, f). Bei Biomasseanlagen können Konflikte die Einhaltung von Klimaschutzzielen betreffen oder ethische Bedenken, potenzielle Lebensmittel (z. B. Mais oder Getreide wie Gerste, Roggen, Weizen) in Energie umzuwandeln (Ziekow et al. 2014b). Die werteorientierte Kritik ist hier vor allem der Tank-oder-Teller-Diskussion geschuldet und nicht der Anlage an sich; Möglichkeiten der Konfliktminderung sind 
daher kaum möglich. Gleiches gilt für Geothermievorhaben und die Wertekritik der technologischen Nutzung der Erdwärme der Erdrinde. Bei Höchstspannungsleitungen entstehen Wertekonflikte häufig durch Landschaftseingriffe. Eine enge, sachliche Zusammenarbeit aller Beteiligten kann helfen, akzeptable Alternativen der Trassenausgestaltung zu entwickeln, z.B. wie Masten unauffälliger und damit verträglicher ins Landschaftsbild integriert oder ob Erdkabel genutzt werden können (Ziekow et al. 2014c). Gleiches gilt für Windenergieanlagen (Verhinderung der „Verspargelung“ der Landschaft) und Pumpspeicherkraftwerke, bei denen ebenfalls das Landschaftsbild verändert wird (Ziekow et al. 2014d, f). Bei Pumpspeicherkraftwerken bezieht sich die werteorientierte Kritik häufig auf die Größe des Kraftwerks und den prognostizierten Speicherbedarf. Ein regionales Gesamtkonzept oder ein gemeinsam abgestimmtes Untersuchungsdesign kann das Konfliktpotential senken.

\subsection{Sozialer Konflikt}

Bei einem sozialen Konflikt handelt es sich um eine eigenständige, latente, bereits bestehende Konfliktsituation, die durch den Streit um das Vorhaben forciert wird (Ziekow et al. 2014b). Bei bestehenden Konfliktsituationen kann es z.B. um parteipolitische Auseinandersetzungen (etwa im Kontext bevorstehender Wahlen) oder um subjektiv empfundene Benachteiligungen (z. B. zwischen Gemeinden) gehen. Diese schon bestehende Konfliktsituation stellt den Hauptauslöser des Konflikts dar. Während die eine Partei versucht die Vorteile des Vorhabens zu nutzen, um eigene Interessen während des Konflikts durchzusetzen, versucht die andere Partei möglicherweise die Risiken des Vorhabens zu nutzen. Daher ist es wichtig, den ursächlichen Problemkern zu finden und zu bearbeiten und sich nicht nur auf den Konflikt beim Vorhaben zu beschäftigen.

\subsection{Wissenskonflikt}

Wissenskonflikte basieren auf divergenten wissenschaftlichen (Experten-)Meinungen. Fachfragen werden aufgrund unterschiedlicher Wissensansätze und -grundlagen unterschiedlich bewertet (Ziekow et al. 2014b). Die daraus resultierenden differierenden Auffassungen, z.B. zu potenziellen Risiken, können zu Auseinandersetzungen um die Wahrheit und Validität des Wissens bzw. Nichtwissens über Technologien führen (Bornemann und Saretzki 2018). Bei Höchstspannungsanlagen können Wissenskonflikte die Einschätzung gesundheitlicher Folgen durch elektromagnetische Strahlung betreffen, bei Windenergieanlagen die Einschätzung der Wirkung von Infraschall und dessen Auswirkungen auf Fledermäuse (Ziekow et al. 2014c, f). Bei Tiefen-Geothermie können sich Wissenskonflikte ergeben bei der Bewertung, ob ihre Nutzung die Erdbebengefahr in Gefährdungsgebieten erhöht oder in Bezug auf die Ökobilanz der Anlagen (Ziekow et al. 2014e). Bei Pumpspeicherkraftwerken können Konflikte hinsichtlich der Standsicherheit von Staumauern und der Auswirkungen auf das Grundwasser bestehen; bei Biomasseanlagen sind sich Experten uneinig, ob Geruchsbelastungen durch die Anlagennutzung auftreten (Ziekow et al. 2014b, d). Die Menge und Unübersichtlichkeit erstellter Expertengutachten und -gegengutachten erschwert häufig Außenstehenden und Betroffenen, die Sachlage korrekt einzuschätzen und zu bewerten. Die langjährige regionale Erfahrung von Anwohnern kann Experten helfen, ihr Wissen zu erweitern und so zu neuen Einsichten zu gelangen.

\section{Konfliktkommunikation}

Es gibt verschiedene Formen und Vorgehensweisen der Konfliktkommunikation. Zu ihnen gehören die Bearbeitung in Beteiligungsverfahren und (damit verbundene) Kommunikationsangebote. Die Möglichkeiten der (kommunikativen) Bearbeitung differieren - so etwa Ziekow et al. (2014a) - abhängig vom Konflikttyp und der Beteiligungsstufe. Unterschiede betreffen die Beteiligungsformate, die zur Konfliktbewältigung eingesetzt werden können, die zu lösenden kommunikativen Aufgaben und die Anforderungen an Kommunikationsversuche.

\subsection{Konfliktanalyse}

Die empirische Konfliktanalyse unterscheidet nach Saretzki (2010) drei Analysearten: gegenstandsbezogene (sachliche Dimension), akteursbezogene (soziale Dimension) und regelungsorientierte (prozedurale Dimension) Konfliktanalyse. Die sachliche Dimension bezieht sich auf die Klärung des Konfliktgegenstands (z.B. Anlagenstandort), die soziale Dimension auf die Akteure bzw. Akteurskonstellationen und Strategien, die prozedurale Dimension umfasst mögliche Konfliktbearbeitungsverfahren, die zu einer Konfliktlösung beitragen können (ebd.) Angesichts der Praxis, in Konfliktsituationen sofort aktiv zu werden, empfiehlt Schwarz (1990), beim Auftreten eines Konflikts diesen zunächst zu analysieren und darauf basierend den Lösungsansatz zu entwickeln, um ein überstürztes, unüberlegtes Handeln zu vermeiden. Um ein aussagekräftiges Bild zur Konfliktsituation zu erhalten, sollten alle drei Dimensionen der Analyse einbezogen werden. Ziekow et al. (2014a) beschreiben ein Konzept für die Durchführung konfliktmindernder Verfahren. Sind Risiken vorhanden, empfehlen sie im Falle von Infrastrukturvorhaben (vor dem Auftreten eines Konflikts) zunächst ein Scoping, zu dem die Genehmigungsbehörde u. a. den Vorhabenträger und die betroffene(n) Kommune(n) 
Abb. 2 Möglicher Ablauf vom Auftreten eines Risikos über einen Konflikt bis zur Krise (eigene Darstellung)

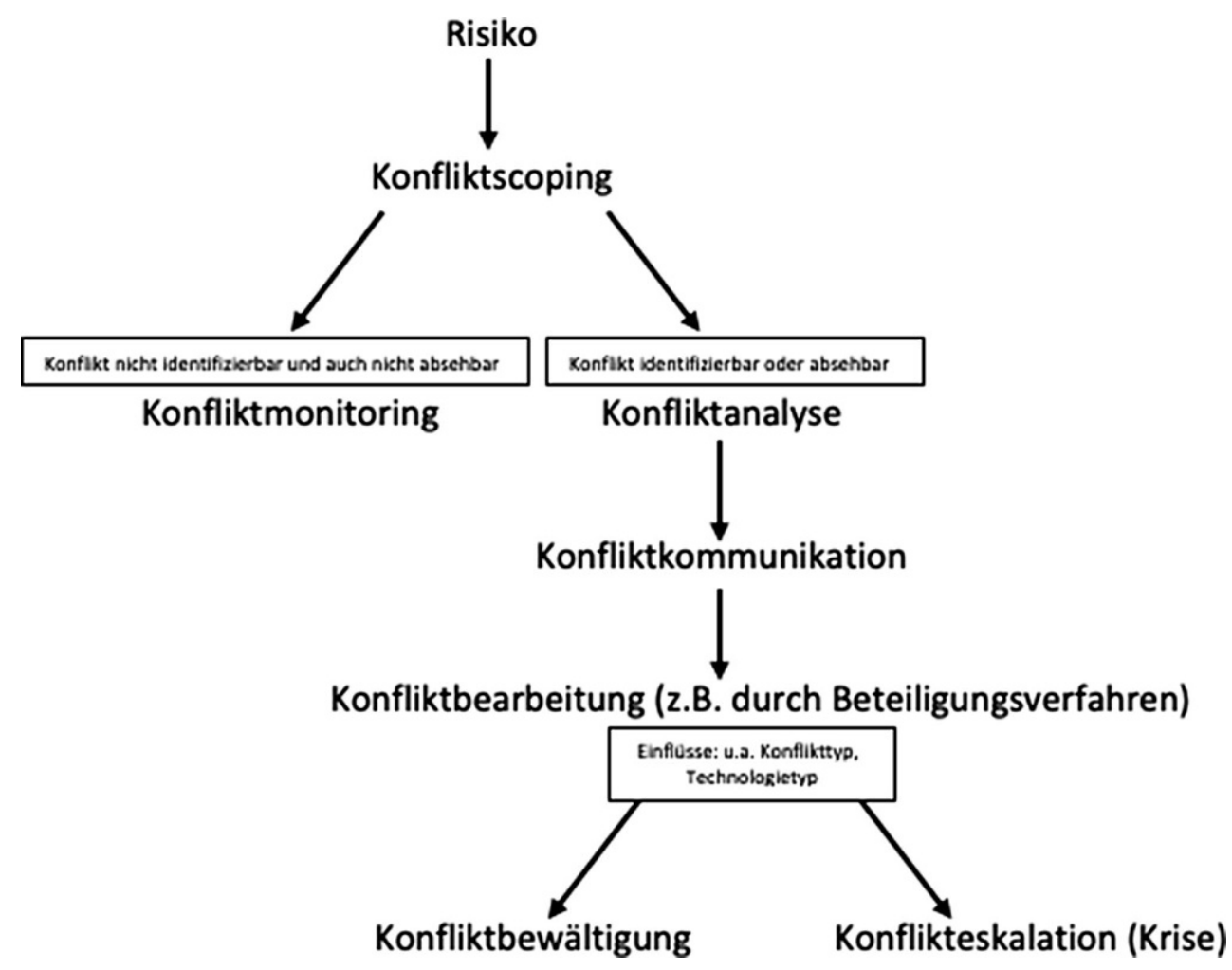

einlädt. Gemeinsam werden Zuständigkeiten (Beobachtung, ggf. konfliktmindernde Verfahren) festgelegt und in einem zweiten Prüfschritt eine Frühdiagnose durchgeführt. Ziel der Frühdiagnose ist, das Konfliktpotential und die Konfliktwahrscheinlichkeit abzuschätzen sowie zu klären, ob (informelle) konfliktmindernde Verfahren nötig sind. Wird kein Konflikt identifiziert bzw. kann der Eintritt eines Konflikts nicht prognostiziert werden, genügt ein Monitoring der Situation (Barth et al. 2018). Wird ein Konflikt identifiziert, muss er analysiert und mögliche Eskalationsstufen betrachtet werden, um abschätzen zu können, wie stark der Konflikt ausfallen wird (vgl. dazu auch Glasl 2011). Teil der Analyse ist die Festlegung und Ausgestaltung der konfliktmindernden kommunikativen Maßnahmen. Die Behörde sollte bei der Ausgestaltung alle Beteiligten, Themen, Ziele und sonstige Rahmenbedingungen betrachten. Anschließend folgt die Konfliktbearbeitung (Abschn. 5.2). Das Ergebnis ist entweder eine Konfliktbewältigung und -lösung oder eine Eskalation des Konfliktes, die in einer Krise gipfelt (siehe Abb. 2).

\subsection{Konfliktbearbeitung in formellen und informellen Beteiligungsverfahren}

Treten Konflikte auf, können Beteiligungsverfahren diese mindern. Brettschneider (2016a) geht davon aus, dass Öffentlichkeitsbeteiligung auf drei Stufen der Beteiligung stattfinden muss: Informations-, Konsultations- sowie die
Kooperationsstufe (Stufe der Mitgestaltung). Ziekow et al. (2014b, c, d, e, f) betrachten dies differenziert abhängig vom Konflikttyp (vgl. Abschn. 5.3).

Da sich Konflikte, Proteste oder kontroverse mediale Debatten häufig zuerst auf lokaler Ebene bilden, bspw. weil sich Gruppen zusammenschließen, die sich gut kennen und in vielen Fragen dieselben Ansichten teilen, empfehlen Studien und Organisationen, Stakeholdergruppen in die Projektplanung einzubeziehen (Möglichkeit der Partizipation), um eine gemeinsame, sozialverträgliche Lösung $\mathrm{zu}$ finden (u.a. Cotton und Devine-Wright 2012; Renn 2015; Mast und Stehle 2016; Hellmuth und Jakobs 2019a).

Die kommunikative Bearbeitung von Konfliktsituationen wird von den meisten Autoren bezogen auf Verfahren der Öffentlichkeitsbeteiligung (formell/informell/finanziell) diskutiert. Es wird darauf verwiesen, dass nicht jeder Konflikt zu jedem Zeitpunkt und in jedem Beteiligungsverfahren gelöst werden kann (Eltrop et al. 2014; Bock et al. 2017). Die Angebote der Öffentlichkeitsbeteiligung müssen auf das Konfliktpotential eines Vorhabens abgestimmt werden (je höher das Potenzial, desto höher der Grad der Beteiligung).

Vor allem Genehmigungsbehörden müssen bei Infrastrukturvorhaben im Rahmen von Planungs- und Genehmigungsprozessen eine rechtlich maßgebliche und bindende formelle Öffentlichkeitsbeteiligung durchführen. Ziekow et al. (2014a) gehen davon aus, dass bei konfliktlosen Vorhaben formelle Bürgerbeteiligungsverfahren ausreichen. 
Da Infrastrukturvorhaben jedoch selten konfliktlos verlaufen und mehr als ein Drittel der Haushalte formelle Beteiligungsangebote als unzureichend bewerten (Fuhrberg und Umansky 2016), sollten insbesondere großtechnische Vorhaben frühzeitig durch informelle Beteiligungsangebote begleitet und ergänzt werden oder versucht werden das Konfliktpotential durch (neue) finanzielle Beteiligungsmöglichkeiten für Bürger (z. B. in Form von Genossenschaften) zu senken. Dabei müssen die Unterschiede formeller und informeller Beteiligung erklärt und beide Verfahren miteinander verzahnt werden.

In formellen Genehmigungsverfahren werden gesetzlich relevante Themen behandelt; informelle Verfahren sind rechtlich nicht vorgesehen und adressieren rechtlich nicht geregelte Themen. Gesetzlich relevant sind u.a. gesundheitliche Fragen (z.B. Lärm, Schallemissionen), Themen des Natur- und Artenschutzes (z.B. Störung des Brutverhaltens durch Leitungen) und speziellere Themen wie Grundwasserschutz, Erdbebengefährdung oder Bodenhebungen/-senkungen (Ziekow et al. 2014b, c, d, e, f). Im Gegensatz zur formellen Beteiligung wird die informelle Beteiligung überwiegend vom Vorhabenträger organisiert (Möller und Zdiara 2017); sie geht über die gesetzlichen Vorgaben hinaus und beteiligt Bürger an Planungs- und Genehmigungsprozessen der Infrastrukturvorhaben. Themen können z.B. Immobilienwertverluste sein, (über gesetzliche Grenzwerte hinausgehende) Gesundheitsfragen, Auswirkungen auf Erholung, Tourismus und Landschaftsbild (z. B. durch den steigenden Anbaubedarf für Mais) (Ziekow et al. 2014b, c, d, e, f) oder sonstige Individualinteressen Betroffener (Roßnagel et al. 2014).

Für die informelle Beteiligung bieten sich dialogische Formate an, die auf Personen zugeschnitten sind, die Angebote formeller Beteiligung eher nicht nutzen. Informelle Prozesse intendieren Akzeptanz für das Genehmigungsverfahren (nicht des geplanten Vorhabens) sowie eine als fair empfundene Entscheidung. Das Ergebnis von Konfliktminderungsprozessen ist im besten Fall eine Einigung, die den Konflikt löst. Dabei soll Beteiligung durch Informieren Transparenz schaffen, das Projekt bekannt machen und den Planungsprozess erklären. Wenn die Situation mehr als Informieren erfordert, sollen - um zu einem Konsens zu gelangen - der Austausch durch Konsultationsprozesse gefördert, Vorschläge und Ideen diskutiert sowie Bedenken ausgeräumt werden. Vielfach werden dialogorientierte Veranstaltungen, wie Stakeholderdialoge oder runde Tische, vorgeschlagen (Geis 2010; Riede 2013; Schönrock 2016). Auf der nächsten Stufe werden gemeinsam strukturiert Empfehlungen erarbeitet (Trias von Interagieren, Mitbestimmen, Mitgestalten) mit dem Ziel, Fakten zu klären und so Konflikte zu entschärfen bzw. zu lösen. Die letzte Stufe greift, wenn sich mindestens zwei Parteien mit gegensätzlichen Standpunkten gegenüberstehen und ein Kon- sens ohne externe Unterstützung nicht (mehr) erreicht wird. Das Ziel ist eine einvernehmliche Einigung der beteiligten Parteien (z.B. durch ein Mediationsverfahren). Der Prozess wird durch überparteiliche und unparteiische Mediatoren und/oder Moderatoren begleitet, die gezielt Verhandlungstaktiken einsetzen. Wesentlich ist, „dass die Verantwortung für die eigenständige Entwicklung einer Lösung bei den streitenden Parteien liegt“" (Nanz und Fritsche 2012, S. 63). Teil des Prozesses ist eine garantierte Ergebnisoffenheit, die freiwillige Teilnahme aller Konfliktparteien und die Verpflichtung zu Vertraulichkeit. Ergebnis einer erfolgreichen Mediation ist z.B. eine außergerichtlich erfolgte, aber dennoch verbindliche Einigung in Form einer Vereinbarung oder eines öffentlich-rechtlichen Vertrags, die von allen Konfliktparteien akzeptiert oder zumindest toleriert wird (siehe auch Wachholz 2008). Sollte auch dies nicht zur Einigung führen, müssen dritte Parteien eine Entscheidung herbeiführen, z. B. durch Schlichtungsverfahren oder gerichtliche Entscheidungen (Riede 2013). Nachteile informeller Verfahren betreffen vor allem den zeitlichen und finanziellen Mehraufwand ihrer Organisation, Planung und Durchführung (Albrecht et al. 2013). Ein Problem formeller Verfahren ist, dass die Möglichkeiten der Einflussnahme und zur Minimierung der Konfliktpotentiale im Frühstadium der Projektplanung am höchsten sind, das Interesse der Bevölkerung jedoch am geringsten (Partizipationsparadox) (Albrecht et al. 2013; Grünwald et al. 2015; Hänlein und El Alaoui 2015); die Möglichkeiten der Beteiligung werden größtenteils erst im Genehmigungsverfahren und mit sinkender Abstraktion des Projekts wahrgenommen (Roßnagel et al. 2014). Gelingt es, in informellen Verfahren frühzeitig das (öffentliche) Interesse für das Projekt zu erhöhen und Stakeholdergruppen zu erreichen, kann sich dies positiv auf das formelle Verfahren auswirken und dem Partizipationsparadox entgegenwirken.

Nach Müller (2020) erhöht sich die Wahrscheinlichkeit der Teilnahme an Formaten der Bürgerbeteiligung durch verfügbare Ressourcen (Geld, Zeit, Bildung), psychologische Motivationsfaktoren (politisches Interesse, wahrgenommene Risiken, Vorteile des Projekts, eigene Betroffenheit), die Mitgliedschaft in Bürgerinitiativen und das Sprechen über Energieinfrastrukturprojekte. Die Wahrscheinlichkeit der Akzeptanz für Erneuerbare-EnergienAnlagen steigt, wenn bereits Erfahrungen mit Infrastrukturprojekten im Lebensumfeld vorliegen (Wunderlich 2012, S. 9f.) oder wenn die Konsequenzen einer Technologie geringer als deren Nutzen eingeschätzt werden bzw. positiv sind.

Eine interessante Variante bieten digitale Beteiligungsmöglichkeiten. Die Rahmenbedingungen wurden infolge der COVID-19-Pandemie und damit einhergehender Kontaktverbote geschaffen, die die Umsetzung der Öffentlichkeitsbeteiligung bei Infrastrukturprojekten erschweren. Am 
15. Mai 2020 hat der Bundesrat (2020; Beschluss 247/20) dem vom Bundestag verabschiedeten „Gesetz zur Sicherstellung ordnungsgemäßer Planungs- und Genehmigungsverfahren während der COVID-19-Pandemie“ (Planungssicherstellungsgesetz) zugestimmt. Es soll u.a. die Konfliktbearbeitung bei physischer Distanz unterstützen. Formelle Verfahren der Öffentlichkeitsbeteiligung werden für einen begrenzten Zeitraum um digitale Möglichkeiten erweitert. Ist z. B. eine öffentliche Bekanntmachung vorgesehen, darf diese (neben der verpflichtenden Veröffentlichung in einem amtlichen Veröffentlichungsblatt oder einer Lokalzeitung) online erfolgen; für die Durchführung eines Erörterungstermins genügt eine Online-Konsultation. In informellen Beteiligungskontexten machte der Übertragungsnetzbetreiber 50hertz erste Erfahrungen mit Online-Marktplätzen und Telefonsprechstunden, die laut eigener Aussage hinsichtlich Quantität und Qualität vergleichbar gut angenommen wurden wie frühere Vor-Ort-Dialoge (50hertz 2020; demgegenüber Hellmuth und Jakobs 2019a, b zu digitalen Beteiligungsformaten und ihre Ablehnung durch die Stakeholdergruppe Landwirte).

\subsection{Konflikttypbezogene Kommunikation}

Im hier diskutierten Zusammenhang sind insbesondere Arbeiten interessant, die sich zu Konfliktkommunikation bezogen auf Konflikttypen äußern. In der ausgewerteten Literatur äußern sich dazu nur sieben Texte ausführlicher - ein allgemeiner und fünf vorhabenspezifische Leitfäden (Ziekow et al. 2014a, b, c, d, e, f) sowie die Publikation zu Mobilfunksendeanlagen von Benighaus et al. (2010).

Die Leitfäden von Ziekow et al. (2014a, b, c, d, e, f) beziehen sich auf in Kap. 4 vorgestellten Konflikttypen Verteilungskonflikt, Wertekonflikt, sozialer Konflikt und Wissenskonflikt. Die Autoren äußern sich zu relevanten kommunikativen Formaten der Konfliktbewältigung, zu Einflussgrößen auf die Wahl von Format und Thema sowie zu beteiligten Akteuren. Wichtig für die Formatauswahl eines Beteiligungsverfahrens sei die jeweilige Beteiligungsstufe und der Konflikttyp, relevant für die Themenauswahl sei dagegen die Technologie des Infrastrukturvorhabens. Die Autoren begrenzen den Zusammenhang von Konflikttyp und Art der kommunikativen Bearbeitung auf die Beteiligungsstufen Konsultation, Kooperation und Einigung. Im Gegensatz zu anderen Autoren umfasst der Ansatz von Ziekow et al. (2014a) mit der Stufe der Einigung eine vierte Beteiligungsstufe. Sie umfasst Verfahren der Einigungssuche und weicht von der normalerweise in der Partizipationsforschung verwendeten vierten Stufe eigenverantwortliches Handeln bzw. Selbstbestimmung von Schweizer-Ries et al. (2010) ab. Maßnahmen auf der Beteiligungsstufe Informieren gelten dagegen als konflikttypübergreifend (siehe Abschn. 5.4). Die Themen variieren ab- hängig vom Technologietyp (siehe oben, Abschn. 4.2-4.5) wie auch die Stakeholdergruppen. Die Bandbreite beteiligter Akteure reicht von Nachbarn/Anliegern/Anwohnern, Nutznießern/Leidtragenden, Gewerbetreibenden, Landwirten, Verbänden und Produzenten bis zu Hotels. Ein und dieselbe Stakeholdergruppe kann von unterschiedlichen Konflikttypen betroffen sein. Im Folgenden werden Befunde bezogen auf Konflikttypen referiert.

Verteilungskonflikt: Ziekow et al. (2014b, c, d, e, f) nennen auf der Konsultationsstufe als wichtiges Konfliktbearbeitungsformat das frühzeitige Gespräch mit betroffenen Personen. Die Zielgruppen variieren je nach Technologietyp: Bei Verteilungskonflikten im Kontext des Ausbaus von Höchstspannungsleitungen sollten vor allem Landwirte, Anwohner oder andere Grundstücksinhaber als potenzielle Nutznießer oder Leidtragende einer möglichen Trassenführung einbezogen werden (Ziekow et al. 2014c). Beim Bau von Pumpspeicherkraftwerken hingegen sollten Zielgruppen adressiert werden, die in der Nähe von Oberund Unterbecken leben bzw. tätig sind (Bürger, Gewerbetreibende, Landwirte) (Ziekow et al. 2014d). Wichtige Adressaten im Kontext von Biomasseanlagen sind die potenziellen Produzenten und deren Anwohner, bei Windenergieanlagen Landwirte im Einzugsgebiet der Anlage sowie ggf. Umwelt- und Naturschutzverbände, Hotels oder Tourismusverbände (Ziekow et al. 2014b, f). Andere Studien bestätigen die Relevanz persönlicher Gespräche, sie werden von Betroffenen bei Konflikten präferiert (z.B. Hellmuth und Jakobs 2019a, b). Als weiteres Format der kommunikativen Bearbeitung von Verteilungskonflikten wird das Format Frage- und Antwortrunde genannt - Bürger fragen, Experten antworten (Ziekow et al. 2014b, c, d, e, f). Ziel der Veranstaltung ist es, Bürgern zu ermöglichen, in einer unabhängig geleiteten Veranstaltung mit Fachleuten ins Gespräch zu kommen, wobei der Fokus auf Vorhabenfolgen, z. B. für die Immobilienwertentwicklung, liegt.

Auf Kooperationsstufe wird (technologie-unabhängig) ein Planungsworkshop mit potenziell Betroffenen empfohlen, der möglichst früh durchgeführt werden sollte. Das gemeinsame Erarbeiten von Positionen sollte aktiv durch Anschauungsmaterialien, die Inhalte visualisieren (Pläne, Flurkarten, etc.), unterstützt und vereinfacht werden. Ziel ist eine Planungsoptimierung (Ziekow et al. 2014b, c, d, e, f), bei Höchstspannungsleitungen auch das Erarbeiten alternativer Trassenführungen (Ziekow et al. 2014c). Der Vorhabenträger sollte offen und kompromissbereit in die Veranstaltung gehen. Um Negativfolgen zu vermeiden und eine einvernehmliche Lösung zu erreichen, werden Runde Tische für die Erarbeitung von Vorschlägen empfohlen. Wenn alle an einer Lösung interessiert sind, die bisherigen Schritte aber nicht zu dieser führen, kann ein klassisches Mediationsverfahren durchgeführt werden, um in struktu- 
Tab. 1 Zusammenhang zwischen Konflikttyp, Beteiligungsstufe und -format (Ziekow et al. 2014b, S. 23)

\begin{tabular}{|c|c|c|c|c|}
\hline & Verteilungskonflikt & Wertekonflikt & Sozialer Konflikt & Wissenskonflikt \\
\hline Information & \multicolumn{4}{|c|}{$\begin{array}{l}\text { Beim Informieren gelten die Formate konflikttyp-übergreifend, z. B. Informationsveranstaltung, Postwurfsendung, Broschüre/ } \\
\text { Flyer, digitale Kanäle der Informationsbereitstellung wie Internetangebote }\end{array}$} \\
\hline Konsultation & $\begin{array}{l}\text { Gespräch mit Betroffenen } \\
\text { Bürger fragen, Experten } \\
\text { antworten }\end{array}$ & $\begin{array}{l}\text { Früher Workshop mit Kriti- } \\
\text { kern } \\
\text { Öffentliche Veranstaltung } \\
\text { mit Speakers' Corner }\end{array}$ & Regionaler Wirtschafts-Workshop & Experten-Hearing \\
\hline Kooperation & $\begin{array}{l}\text { Planungsworkshop } \\
\text { Runder Tisch }\end{array}$ & $\begin{array}{l}\text { Verfahren auf diesen Stufen } \\
\text { nicht mehr sinnvoll }\end{array}$ & Bürger-Jury & $\begin{array}{l}\text { Fachworkshop } \\
\text { Fachgutachten }\end{array}$ \\
\hline Einigung & Klassische Mediation & & $\begin{array}{l}\text { Kein Format, sondern eigentliche, } \\
\text { verdeckte Ursache aufdecken }\end{array}$ & Data-Mediation \\
\hline
\end{tabular}

rierten Verhandlungsprozessen doch noch eine Einigung zu erreichen (Ziekow et al. 2014b, c, d, e, f).

Wertekonflikt: Als konfliktminderndes Format auf Konsultationsstufe wird ein (frühzeitiger) Workshop mit wertorientierten Kritikern empfohlen, die Umwelt-, NaturschutzBauernverbänden oder lokalen zivilgesellschaftlichen Organisationen angehören können (Ziekow et al. 2014b, c, d, e, f). Alle Beteiligten sollen kollektiv eine Optimierung der Anlage bzw. der Stromleitung erarbeiten. Ein Format, das Wertekonflikte mildern und Emotionalisierung verhindern kann, ist eine Veranstaltung mit Speakers' Corner, in denen jede Konfliktpartei ihren eigenen Standpunkt darlegen darf. Bogner und Menz (2010) nennen (konkret für Konflikte der Biomedizin) weitere Mittel wie das Gründen von EthikKommissionen, Bürgerkonferenzen und Experten-LaienDebatten. Über die Konsultation hinausgehende Beteiligung ist bei einem Wertekonflikt nur schwer realisierbar und nicht mehr sinnvoll, da der Aufwand zu groß und der Ertrag in der Regel zu gering ist (Ziekow et al. 2014b, c, d, e, f).

Sozialer Konflikt: Bei einem sozialen Konflikt wird auf der Konsultationsstufe empfohlen, einen SzenarioWorkshop in einem regional wichtigen Wirtschaftszweig durchzuführen (z.B. Landwirtschaft/Tourismus) (Ziekow et al. 2014b, c, d, e, f). Im Workshop sollen alle beteiligten Stakeholder gemeinsam Perspektiven diskutieren und Aspekte des Vorhabens berücksichtigen. Die Inhalte und Themen des Workshops variieren je nach Technologietyp. Bei Höchstspannungsleitungen kann das auslösende Moment z.B. der Konflikt sein zwischen einem energieintensiven, bereits in der Kritik stehenden Regional-Betrieb, der den Leitungsneubau befürwortet, und Bürgern, die eine Gesundheitsgefährdung durch die Leitung befürchten (Ziekow et al. 2014c). Thema des Workshops sind dann Auswirkungen des Netzausbaus. Bei Geothermievorhaben kann der Ausgangspunkt ein Konflikt sein zwischen alteingesessenen Bürgern, die neue Infrastruktur befürworten, und Zugezogenen, die den ruralen Charakter ihres Wohnortes und dessen Ruhe schätzen (Ziekow et al. 2014e). Bei Pumpspeicherkraftwerken kann es die Sorge sein, dass die Tourismusbranche während der Bauphase in Existenznot gerät, gepaart mit der bereits sinkenden Attraktivität der Region (Ziekow et al. 2014d). Im Workshop wird gemeinsam ein Zukunftskonzept für den regionalen Tourismus erarbeitet, das die veränderte Attraktivität der Region berücksichtigt.

Auf der Kooperationsstufe wird für alle Technologietypen die Einrichtung einer Bürger-Jury (mit repräsentativer Stichprobe der örtlichen Bevölkerung) empfohlen. Aufgabe der Jury ist, an der Konfliktlösung mitzuwirken und Empfehlungen zu erarbeiten.

Wissenskonflikt: Als hilfreiches konfliktminderndes Konsultationsverfahren nennen Ziekow et al. (2014b, c, d, e, f) das Experten-Hearing. Im Hearing wird das Spektrum fachlicher Meinungen aufgezeigt und verdeutlicht, wo der Bereich des Nicht-Wissens liegt. Neue Erkenntnisse, die sich aus den Gutachten ergeben (z.B. durch veraltete Berechnungsmethoden) müssen im Rahmen der Amtsermittlungspflicht der Genehmigungsbehörde (bei Kenntnis) berücksichtigt werden.

Auf Kooperationsstufe empfehlen Ziekow et al. (2014b, c, d, e, f) einen Fachworkshop, bei dem ein Vorschlag des Vorhabenträgers anhand von Experten unterschiedlicher Fachrichtungen auf fachliche Robustheit geprüft wird. Ergebnis der Prüfung ist optimalerweise eine gemeinsame Wissensgrundlage, auf die sich die Experten einigen. Wichtiger, als alle Fragen möglichst granular zu behandeln, ist eine Einigung in Grundsatzfragen. Ein weiteres Format zur Konfliktminderung sind Sachverständigengutachten oder die Qualitätssicherung bestehender Gutachten durch unabhängige Experten (ebd.). Die Gutachten müssen bei Kenntnis im Rahmen der Amtsermittlungspflicht berücksichtigt werden. Eine Einigung kann auch durch eine Übereinkunft über den zugrunde gelegten Ausgangs-Sachverhalt erzielt werden (verbindliche Sachverhaltsvereinbarung), an die sich die Konfliktparteien halten müssen (sog. Data-Mediation) (ebd.).

Ziekow et al. (2014b, c, d, e, f) geben einen Überblick, der Zusammenhänge zwischen Konflikttyp, Beteiligungsstufe und Format darstellt (vgl. Tab. 1). Die Übersicht ver- 
deutlicht, dass Interventionen zur Bearbeitung von Konflikten - in diesem Fall die strategische Wahl von Formaten - deutlich variieren je nach Konflikttyp. Dies gilt, wie oben dargestellt, weitgehend für die Beteiligungsstufen Konsultation und Kooperation, nicht jedoch für die Stufen Information und Einigung. Der Impact des Konflikttyps wiegt schwerer als der der Technologie, um die es geht. Die Autoren schlagen die unten abgebildeten Maßnahmen unabhängig vom Technologietyp vor, sie diskutieren zugleich die Eignung dieser Formate am Beispiel verschiedener Technologietypen, z. B. in Ziekow et al. (2014d) für Pumpspeicherkraftwerke oder in Ziekow et al. (2014e) für Geothermie. Der Impact der Technologie zeigt sich weniger bei den Formaten, als vielmehr - wie oben erwähnt - in den zu bearbeitenden Themen und in den zu adressierenden Akteursgruppen.

Benighaus et al. (2010) empfehlen ein kombiniertes Dialogverfahren. Dieses besteht aus einer vorgelagerten Informationsveranstaltung, die vor allem Wissenskonflikte begrenzen soll, und einem Mediationsverfahren, das mit einer Diskussions- und Bewertungsrunde kombiniert wird.

\subsection{Kommunikative Gestaltungsaspekte}

Insgesamt fällt auf, dass in der Literatur zu Konflikten und Konfliktkommunikation zwar konstatiert wird, dass Konfliktkommunikation ein wichtiges Mittel der Konfliktbearbeitung ist bzw. Konflikte kommunikativ bearbeitet werden müssen. Es wird aber kaum ausgeführt, wie die Kommunikation aussehen soll, welchen Kriterien sie genügen muss oder wo die Herausforderungen der Gestaltung liegen. Die Ausführungen zum Wie beschränken sich meistens auf das Nennen unterschiedlicher Akteursgruppen, potenziell relevanter Themen und von Formaten. Es gibt nur wenige Autoren, die sich detailliert zur Gestaltung von Konfliktkommunikation äußern. Hier lohnt es sich, den Blick auf die Literatur zur kommunikativen Begleitung von Infrastrukturprojekten zu erweitern. Sie bietet verschiedene Ansätze, die gewinnbringend für das Thema Konfliktkommunikation sind. Im Folgenden werden ausgewählte kommunikative Verfahren zur Bearbeitung von Konflikten und Aspekte ihrer Gestaltung angesprochen. Sie umfassen vor allem Formen monologischer und dialogischer (eins- und zweiseitiger Kommunikation) sowie persönlicher und massenmedialer Kommunikation.

In verschiedenen Zusammenhängen wird betont, dass Informieren (einseitige Kommunikation) eine wichtige Aufgabe und ein wichtiges Mittel zum Vermeiden und Bearbeiten von Konflikten ist (u. a. Benighaus et al. 2010; Hampel und Torgersen 2010; Jobst 2010; Kriegel 2011; Hübner und Pohl 2014; Ziekow et al. 2014a; Renn 2015). Dies kann verschiedene Gründe haben. Neue Technologien sind z. B. in der Bevölkerung häufig weitgehend unbekannt (In- formationsdefizit). Kommt es zur Konfrontation mit diesen Technologien, z.B. im Falle ihrer Anwendung in Projekten, gründen wahrgenommene Risiken häufig nicht auf einer faktenbasierten Auseinandersetzung, sondern auf Vermutungen (Borg et al. 2018 zu Tiefen-Geothermie). Als wesentlicher Bestandteil einer gezielten Konfliktminimierung gilt daher das (umfassende und idealerweise proaktive) Bereitstellen und Vermitteln von Informationen (mündlich, schriftlich, bildlich) (Kriegel 2011; Albrecht et al. 2013; Renn 2015). Borg et al. (2018) sehen die Verfügbarkeit von Information und ihre adressatengerechte Aufbereitung als Voraussetzung für gute Kommunikation und wichtigen Bestandteil von Kommunikationsstrategien. Informationen sollten mit verschiedenen Medien und unterschiedlichen Visualisierungsformen dargestellt werden, um möglichst viele (potenzielle) Zielgruppen (frühestmöglich und niedrigschwellig) zu erreichen (Brettschneider 2011; 2012; Renn 2015; Schmalz 2019). Fehrentz (2020) nennt als Positivbeispiel die Kommunikation von ThyssenKrupp zu ihrem Testturm für Hochgeschwindigkeitsaufzüge in Rottweil, die u.a. Visualisierungen und Simulationen als Mittel der Veranschaulichung nutzt. Neben dem Nutzen eines Projekts bzw. einer Technologie sollten mögliche Negativfolgen multidimensional und transparent beschrieben werden (vgl. Hübner und Pohl 2014, S. 8; Reimer et al. 2015, S. 4). Dabei geht es nicht nur um die Vermittlung faktischen Wissens. Studien zeigen, dass insbesondere die gefühlte Verfügbarkeit von Wissen zu einem Thema wichtig ist für die Einstellung zu und die Auseinandersetzung mit Technologien und technologischen Vorhaben (Hellmuth und Jakobs 2019a, b, 2020).

In der Literatur werden verschiedene Informationsformate genannt, wie z.B. die klassische Informationsveranstaltung, Postwurfsendungen, Pressemitteilung, Broschüren/ Flyer/Plakate (Hellmuth und Jakobs 2019a, b) oder digitale Kanäle der Informationsbereitstellung wie die ProjektWebsite bzw. andere Internetangebote wie Newsletter, Blog (Ziekow et al. 2014b) oder soziale Medien (z. B. Facebook) (Borg et al. 2018). Nach Ziekow et al. (2014b, c, d, e, f) eignen sich die genannten Informationsformate für alle Konflikt- und Technologietypen. Die Planung von Informationsveranstaltungen sollte zeitliche Restriktionen der Erwerbstätigkeit von Bürgern berücksichtigen. Sie sollten eher abends oder am Wochenende stattfinden und maximal 90 min dauern (Ermisch et al. 2018). Verschiedentlich wird darauf hingewiesen, dass Informieren an sich nicht ausreicht bzw. kein Garant für die gewünschten Effekte ist. Wissen bzw. Informationen führen nicht per se zu (mehr) Akzeptanz, sie können auch Skepsis fördern und bestehende Vertrauensdefizite verstärken (Weitze und Renn 2019). Zum Teil sind Personen, die Konflikte auslösen bzw. tragen, sehr gut über die Sachlage informiert (Kluge 2018). 
Dialogische Verfahren: Nach Weitze und Renn (2019) ist bei Themen, die einen Konflikt auslösen können oder die die Öffentlichkeit direkt betreffen, der Dialog (zweiseitige Kommunikation) die angemessene Art der Kommunikation. Vor allem der Einsatz kollaborativer Planungsansätze und Beteiligungsverfahren kann Konfliktsituationen mindern (Cain und Nelson 2013). Dafür ist es unerlässlich, die besonders betroffenen Gruppen zu identifizieren und sicherzustellen, dass sie erreicht werden. Detaillierte Hilfestellungen zur Integration der Öffentlichkeitsbeteiligung in das Projektmanagement bieten die VDI-Richtlinien 7000 bzw. 7001 (VDI 2014, 2015). Insbesondere auf lokaler Ebene präferieren (direkt und indirekt) betroffene Gruppen die direkte Ansprache und das persönliche Gespräch gegenüber medial vermittelter Kommunikation und unpersönlichen Beteiligungsformaten - auch mögliche Beschwerden sollten im direkten Dialog behandelt werden (Ermisch et al. 2018). Direkter Kontakt und Austausch ermöglichen, Perspektivendivergenzen offenzulegen, auf die Positionen des Partners einzugehen und im offenen Dialog Vertrauen aufzubauen (vgl. Heger 2016; Hellmuth und Jakobs 2019a, b). Eine wesentliche Voraussetzung ist die Fähigkeit und der Wille, sich auf den anderen einzulassen. Voraussetzung für eine gelungene Kommunikation mit den Bürgern sind u.a. Weiterbildungsangebote für Mitarbeiter, die diese leisten sollen (Roßnagel et al. 2014).

Die kommunikative Begleitung von Projekten und Vorhaben schließt die Beobachtung der zum Gegenstand laufenden Diskurse und die systematische Antizipation potenziell konfliktträchtiger Themen ein. Nach Brettschneider $(2015,2016 a)$ eignet sich dafür das Verfahren der Themenfeldanalyse mit dem Instrument der Themenlandkarte. Themenlandkarten erfassen relevante Themen für einen Gegenstandsbereich (z.B. als Teil der inhaltlich-strategischen Ausrichtung der Projektkommunikation in der Vorplanungsphase von Bau- und Infrastrukturprojekten, VDI 2014). Sie helfen, die Sicht unterschiedlicher Stakeholder auf ein Thema (Projekt/Technologie) einzuordnen und zu gewichten. Relevant für Zwecke der (präventiven) Konfliktbetrachtung sind so genannte Issues (Themen mit einem hohen (kommunikativen) Konfliktpotential), die mit Themenlandkarten frühzeitig zu identifizieren sind und auf die Interventionsmöglichkeiten angewendet werden können. Kommunikationsstrategien sollten nicht nur Themenfeldanalysen enthalten, sondern auch Sprachregelungen (für Kernbotschaften), eine Liste mit häufig gestellten Fragen und Antworten dazu (FAQ), eine Stakeholder-Analyse und einen Maßnahmenplan (Brettschneider und Müller 2020).

Zum Teil werden konkrete Empfehlungen für die Darstellung von Themen in Print- und digitalen Formaten gegeben. Die Art der Darstellung und der Umfang der Themenbehandlung müssen sich am Adressaten orientieren. Berichte für Presseorgane z.B. müssen bestimmte Vorgaben ein- halten (Ziekow et al. 2014b). Sie sollen nicht länger als eine Seite sein (Weber und Brian 2014 empfehlen etwa acht Absätze), das Thema kontextualisieren und Fakten verbal wie auch visuell darstellen. Im Falle von Online-Kanälen geht es darum, quantitativ angemessen zu informieren und Inhalte wohlstrukturiert anzubieten. Die Startseite sollte nicht zu textlastig sein, sondern Übersichtlichkeit und Zugänglichkeit sichern. Auf Unterseiten können vollständige Unterlagen bereitgestellt und weitergehende Informationen (Ansprechpartner/Ombudsmann, Termine, andere Organisationen etc.) geboten werden. Die Inhalte müssen aktuell sein. Die verbale Berichterstattung sollte durch Bilder und kurze Videos unterstützt werden. Um Einseitigkeit zu vermeiden und Interaktion zu ermöglichen, müssen Online-Formate einen Rückkanal anbieten (Weber und Brian 2014), z. B. in Form eines Kontaktformulars, eines Online-Panels zur Meinungserhebung oder einer Dialogplattform. Bei Veranstaltungen übernehmen diese Funktion Briefkästen, Tafeln oder Flyer mit Rückantwortkarte. Postwurfsendungen, z. B. Broschüren, sollten nicht länger als vier Seiten sein, eine Mindestschriftgröße von $12 \mathrm{pt}$ haben und von einem professionellen Grafikbüro erstellt werden (Ziekow et al. 2014b). Überzeugend gestaltete Formate haben ihren Preis und erfordern entsprechende Budgets für externe Experten, die mit der Umsetzung beauftragt werden.

Visualisierungen: Mit Hilfe von Visualisierungen sollen abstrakte technische oder planerische Sachverhalte in eine visuell leicht erfassbare Form gebracht werden, um die für Laien oft schwer nachvollziehbare Komplexität der Sachverhalte verständlicher zu machen. Für eine exakte und anschauliche Visualisierung einer möglichen Zukunft werden anstatt technischer Skizzen Fotomontagen, Architekturmodelle oder EDV-gestützte Bilder empfohlen (ebd.), geschönte oder manipulative Darstellungen werden von den Bürgern zumeist als solche erkannt und eingestuft (Oppermann und Renn 2019). Alternativ können kostenfreie VorOrt-Führungen mit ausreichend zur Verfügung stehenden Speisen und Getränken durchgeführt werden (Weber und Brian 2014). Ermisch et al. (2018) empfehlen speziell bei Windenergieprojekten eine Videovisualisierung oder eine Darstellung als 3D-Modell, mit der den Bürgern die optischen Auswirkungen (Höhe, Ausrichtung, Entfernungen, Sichtbarkeit) verdeutlicht werden.

Kommunikationsqualität: Für den Erfolg konfliktmindernder Maßnahmen ist die (wahrgenommene) Kommunikationsqualität wichtig (Hellmuth und Jakobs 2019a, b, 2020). Grundlegende Anforderungen richten sich auf Glaubwürdigkeit, Nachvollziehbarkeit, Objektivität/Neutralität, Authentizität, Transparenz und Kommunikation auf Augenhöhe (u. a. Ziekow et al. 2014b; Renn 2015; Reimer et al. 2015). Betroffene wollen das Gefühl haben, dass sie und ihre Interessen ernst genommen werden und ihre Mitsprache erwünscht ist (Demuth und Heiland 2016, 
S. 11f.). Um Konfliktsituationen zu minimieren, müssen kommunikative Angebote die Heterogenität involvierter Gruppen (Alter, Geschlecht, Bildung, Generationszugehörigkeit, Lebensstil, Art und Grad der Betroffenheit, privat/ kollektiv wahrgenommene Kosten, Potenziale und Risiken, kultureller Hintergrund, lokale Verankerung, u.a.) angemessen berücksichtigen (Jakobs 2019), z. B. in Ansprache und Ton oder durch Verständlichkeit sichernde Maßnahmen (z.B. Verzicht auf einen technokratischen Stil oder Fachjargon) (Ziekow et al. 2014c, d, e, f; Brettschneider 2016a; Hellmuth und Jakobs 2019a). Um möglichst viele unterschiedliche Personen in die Kommunikationsprozesse einzubeziehen, sollten introvertierte Persönlichkeiten aktiv durch Kommunikatoren angesprochen werden, z. B. durch aufsuchende Beteiligung (Schmalz 2019). Da Kommunikationsangebote standortbezogen passen müssen, muss geklärt werden, welche Faktoren für eine Region bedeutsam sind und welche Faktoren und Teilaspekte stärker wiegen als andere (Brettschneider 2016a). Fehrentz (2020) empfiehlt, dem Projekt „ein Gesicht zu geben“, indem z.B. Mitarbeiter zu Botschaftern des Projekts gemacht oder porträtiert werden und/oder das Projekt durch Events während der Bauphase erlebbar wird. Bei Veranstaltungen muss auf Feinheiten wie z.B. eine gute Akustik, großzügige Raumbestuhlung und eine neutrale Moderation geachtet werden - schlechte Akustik, überfüllte Räume und fehlende Professionalität können Misstrauen erzeugen oder verstärken (Energieagentur.NRW 2014; Ziekow et al. 2014b). Eine wesentliche Herausforderung ist der konzertierte Einsatz verschiedener Mittel (Hellmuth und Jakobs 2019a). Die Kombination unterschiedlicher Formate erfordert eine sorgfältige inhaltliche und zeitliche Planung sowie Abstimmung.

Die Wahrnehmung der Welt und ihre Interpretation hat immer auch eine emotionale Komponente. Die Einstellung zu Technologien und deren Wahrnehmung wird durch subjektive Bewertungen, Einschätzungen, Werte (Renn 2015; TechnikRadar 2018) und Emotionen (Park et al. 2014) beeinflusst. Meinungsbildung basiert häufig auf Medienberichten und/oder auf Meinungen von Personen bzw. Institutionen, denen Glauben geschenkt wird oder die ähnliche ethische oder moralische Vorstellungen und Wertmaßstäbe haben (Wertsimilarität) (Dürrenberger 2013). Dürrenberger empfiehlt, die Kommunikation konfliktärer Technologien nicht auf akademische Sachinformationen zu beschränken, sondern auch emotionale Komponenten einzubauen, z.B. durch personalisierte Botschaften. Wenn es das Projektbudget erlaubt, können Emotionen durch Imagefilme transportiert werden. Diese sollten zwingend von einem professionellen Anbieter erstellt werden (Weber und Brian 2014).

\section{Fazit}

Die Literatur zu Konfliktkommunikation zeigt Gemeinsamkeiten wie Unterschiede, die im Folgenden kurz und stark verallgemeinert zusammengefasst werden: Die meisten Autoren behandeln Konfliktkommunikation bezogen auf Infrastrukturprojekte allgemein oder auf eine bestimmte Technologie, nicht jedoch bezogen auf einen bestimmten Konflikttyp. Bezüglich des Zusammenhangs von Konflikttyp und Technologietyp bzw. -anwendungsfeld besteht erheblicher Forschungsbedarf. Es herrscht Konsens, dass die Zielgruppen und die zu bearbeitenden Themen abhängig von der Technologie, die den Konflikt auslöst, variieren.

Die meisten Autoren reduzieren ihre Ausführungen zu Konfliktkommunikation auf das Nennen von Anlässen, Zielgruppen und Formaten. Ihnen gemein ist, dass Informieren im direkten Kontakt als wichtiges Mittel gilt, unabhängig vom Konflikt- und Technologietyp. Nur wenige Autoren diskutieren Konfliktkommunikation bezogen auf unterschiedliche Konflikttypen. Sie sehen Zusammenhänge zwischen dem Konflikttyp und der Passung von Formaten für seine kommunikative Bearbeitung ab der Konsultationsstufe. Bei einem Verteilungskonflikt eignet sich z.B. auf Konsultationsebene eine Frage- und Antwortrunde, auf Kooperationsebene Runde Tische; bei einem sozialen Konflikt werden dagegen auf der Konsultationsebene die Durchführung eines Szenario-Workshops, auf Kooperationsebene eine Bürger-Jury empfohlen (vgl. Tab. 1).

Insgesamt zeigt sich ein deutlicher Forschungsbedarf zu passgenauer Konfliktkommunikation wie auch zu Evaluationskriterien für Maßnahmen (Reichweite, Wirkung). Was fehlt, sind unter anderem Studien, die empirisch basiert betrachten, welche Formate sich warum für welchen Zweck eignen bzw. wie sie kombiniert und aufeinander abgestimmt werden sollten. Die Diskussion digitaler Medien bleibt weit hinter den Möglichkeiten zurück, die es aktuell gibt, z.B. durch interaktive Planungstische, die Perspektiven auf den Gegenstand zusammenführen, simulativ Konsequenzen von Entscheidungen visuell erfassbar zeigen und so tragfähige Ausgangspunkte für die Diskussion über den Sinn oder Nicht-Sinn von Alternativen schaffen. Ein weiteres Handlungsfeld ergibt sich mit der Entwicklung von Expertise für Konfliktkommunikation als Bestandteil Technologie und Infrastrukturprojekt begleitender Kommunikationsstrategien. Wünschenswert wären u. a. Angebote in einschlägigen Studiengangsprofilen oder Weiterbildungsangebote für Praktiker. Last but not least wäre Forschung wünschenswert, die alternative Kommunikationsansätze und innovative Formate der kommunikativen Bearbeitung technologie-bezogener Konflikte, die in anderen Ländern praktiziert werden, hinsichtlich ihrer Übertragbarkeit auf den deutschen Raum prüfen bzw. dafür diese adaptierbar machen. 
Die Bearbeitung von Konflikten sowie die Ziele und Instrumente für Konfliktkommunikation müssen klar definiert sein. Ziele, Instrumente und Maßnahmen der Konfliktkommunikation und -bearbeitung sollten natürliche Bestandteile der Gesamtstrategie der kommunikativen Begleitung von Infrastrukturprojekten sein und in diese integriert werden. Diese Forderung erweitert das Kommunikationskonzept für Infrastrukturprojekte nach Brettschneider und Müller (2020). Die Entwicklung eines Konzepts der Öffentlichkeitsbeteiligung, das Konflikte proaktiv antizipiert bzw. als „,natürlichen“ Bestandteil von Demokratie versteht, ermöglicht einen fairen Umgang mit konfligierenden Interessen und das Erarbeiten konstruktiver Lösungen für alle (Roßnagel et al. 2014; Huge und Roßnagel 2018). Die Konzeptentwicklung erfordert nicht nur, Konflikte zu antizipieren, sondern potenzielle Reibungsflächen (Konflikte und deren Themen) Konflikttypen zuzuordnen und darauf aufbauend passgenaue Formate zu entwickeln. Die dazu erforderlichen spezifischen Kompetenzen sollten Gegenstand der oben erwähnten Weiterbildungsangebote sein. Hier besteht erheblicher Handlungsbedarf in der Praxis. Konflikte werden weiterhin fester Bestandteil sozio-technischer Transformationsprozesse sowie der technologischen Entwicklung an sich bleiben. Angesichts einer sich immer stärker ausdifferenzierenden Gesellschaft und eines hochgradig technologisch geprägten Umfelds ist zu erwarten, dass sie in Zukunft zunehmen werden. Mithilfe gelingender Konfliktkommunikation und Öffentlichkeitsbeteiligung können aber auch konfliktbehaftete Infrastrukturprojekte erfolgreich geplant und realisiert werden.

Limitierungen der Studie betreffen zum einen die Auswahl der Literatur (Kombination von Methoden, weitgehende Begrenzung auf deutschsprachige Studien). Möglicherweise wurden dabei weitere für das Thema relevante Arbeiten übersehen. Eine zweite Limitierung betrifft die primär an den Forschungsfragen orientierte Kategorienbildung und die dadurch geleitete Auswertung.

Förderung Das dem Beitrag zugrundeliegende Vorhaben wurde mit Mitteln des Bundesministeriums für Bildung und Forschung unter dem Förderkennzeichen: 03SFK1C0-2 gefördert. Die Verantwortung für den Inhalt dieser Veröffentlichung liegt bei den Autoren.

\section{Literatur}

50hertz (2020) Presseinformation vom 15. Mai 2020. Planungssicherstellungsgesetz ist ein gut ausgewogenes Instrument für Bürgerbeteiligung und Transparenz

Ackermann H, Krämer M, Melsheimer O, Scheffran J (2001) Energienutzung - Konflikte, Potenziale, Szenarien. In: Zoll R (Hrsg) Energiekonflikte - Problemübersicht und empirische Analysen zur Akzeptanz von Windkraftanlagen, Bd. 4. LIT-Verlag, Münster, S $17-95$

Albrecht R, Grüttner A, Lenk T, Lück O, Rottmann O (2013) Optionen moderner Bürgerbeteiligung bei Infrastrukturprojekten. Ableitun- gen für eine verbesserte Beteiligung auf Basis von Erfahrungen und Einstellungen von Bürgern, Kommunen und Unternehmen

Aubert V (1963) Competition and dissensus: two types of conflict and of conflict resolution. J Confl Resolut 7(1):26-42

Barth R, Ewen C, Schütte S, Ziekow J (2018) Konfliktdialog bei der Zulassung von Vorhaben der Energiewende. In: Holstenkamp L, Radtke J (Hrsg) Handbuch Energiewende und Partizipation. Springer VS, Wiesbaden, S 583-595

Bauriedl S (2016) Formen lokaler Governance für eine dezentrale Energiewende. Geogr Z 104:72-91

Becker S, Naumann M (2016) Energiekonflikte nutzen. Wie die Energiewende vor Ort gelingen kann. Leibniz-Institut für Raumbezogene Sozialforschung, Erkner

Becker S, Gailing L, Naumann M (2012) Neue Energielandschaften - neue Akteurslandschaften. Eine Bestandsaufnahme im Land Brandenburg. Rosa-Luxemburg-Stiftung (rls Studien), Berlin

Benighaus C, Kastenholz H, Renn O (2010) Kooperatives Konfliktmanagement für Mobilfunksendeanlagen. In: Feindt PH, Saretzki T (Hrsg) Umwelt- und Technikkonflikte. VS, Wiesbaden, S 275-296

Bock S, Reimann B, Abt J, Lettow M, Vorwerk U (2017) Beteiligungsverfahren bei umweltrelevanten Vorhaben. Abschlussbericht. Umweltbundesamt, Dessau-Roßlau

Bogner A, Menz W (2010) Konfliktlösung durch Dissens? Bioethikkommissionen als Instrument der Bearbeitung von Wertkonflikten. In: Feindt PH, Saretzki T (Hrsg) Umwelt- und Technikkonflikte. VS, Wiesbaden, S 335-353

Borg A, Jakobs E-M, Ziefle M (2018) Kommunikation und Akzeptanz. In: Bauer M, Freeden W, Jacobi H, Neu T (Hrsg) Handbuch Oberflächennahe Geothermie. Springer Spektrum, Berlin, Heidelberg, New York, S 691-713

Bornemann B, Saretzki T (2018) Konfliktfeldanalyse - das Beispiel „Fracking“ in Deutschland. In: Holstenkamp L, Radtke J (Hrsg) Handbuch Energiewende und Partizipation. Springer VS, Wiesbaden, S 563-581

Böschen S (2010) Reflexive Wissenspolitik: die Bewältigung von (Nicht-) Wissenskonflikten als institutionenpolitische Herausforderung. In: Feindt PH, Saretzki T (Hrsg) Umwelt- und Technikkonflikte. VS, Wiesbaden, S 104-122

Brettschneider F (2011) Kommunikation und Meinungsbildung bei Großprojekten. In: Demokratie und Beteiligung. APuZ, Bd. 44-45, S 40-47 (61. Jahrgang)

Brettschneider F (2012) Legitimation durch Kommunikation? Die gesellschaftliche Debatte über Ingenieurprojekte. mining+geo 3:435-439

Brettschneider F (2015) Kommunikation und Öffentlichkeitsbeteiligung in der Energiewende. In: Bundesnetzagentur für Elektrizität, Gas, Telekommunikation, Post und Eisenbahnen (Hrsg) Wissenschaftsdialog 2014. Technologie, Landschaft, Kommunikation, Wirtschaft. Bundesnetzagentur, Bonn, S 13-32

Brettschneider F (2016a) Erfolgsbedingungen für Kommunikation und Bürgerbeteiligung bei Großprojekten. In: Glaab M (Hrsg) Politik mit Bürgern - Politik für Bürger. Springer, Wiesbaden, S 219-238

Brettschneider F (2016b) Widerstände gegen Infrastrukturprojekte. Die Bedeutung von Kommunikationsmanagement für Vorhabenträger und öffentliche Verwaltungen. dms 2018(1):97-118 (11. Jg.)

Brettschneider F, Müller U (2020) Vorhabenträger auf dem Weg zu gesellschaftlich tragfähigen Lösungen. Dialogorientierte Kommunikation bei Bau- und Infrastrukturprojekten. In: Brettschneider F (Hrsg) Bau- und Infrastrukturprojekte. Dialogorientierte Kommunikation als Erfolgsfaktor. Springer Fachmedien, Wiesbaden, S 1-39

Bundesrat (2020) Gesetz zur Sicherstellung ordnungsgemäßer Planungs- und Genehmigungsverfahren während der COVID-19Pandemie (Planungssicherstellungsgesetz - PlanSiG)

Bundesregierung (2019) Krisen verhindern, Konflikte bewältigen, Frieden fördern. Leitlinien der Bundesregierung 
C.A.R.M.E.N. e. V. (2014) Akzeptanz für Erneuerbare Energien. Ein Leitfaden. C.A.R.M.E.N. e. V., Straubing

Cain NL, Nelson HT (2013) What drives opposition to high-voltage transmission lines? Land Use Policy 33:204-213

Conrad J (2010) Ein lokaler Umweltkonflikt in Latenz: Grüne Gentechnik und Entwicklungspfade der Pflanzenbiotechnologie. In: Feindt PH, Saretzki T (Hrsg) Umwelt- und Technikkonflikte. VS, Wiesbaden, S 163-180

Cotton M, Devine-Wright P (2012) Making electricity networks 'visible': Industry actor representations of 'publics' and public engagement in infrastructure planning. Public Underst Sci 21:17-35

Covello VT (1992) Risk communication: An emerging area of health communication research. In: Deetz SA (Hrsg) Communication yearbook, Bd. 15, S 359-373

Dahrendorf R (1961) Gesellschaft und Freiheit: Zur soziologischen Analyse der Gegenwart. Sammlung. Piper, München

Demuth B, Heiland S (2016) Naturverträgliche Energiewende - zwischen allen Stühlen? In: Demuth B, Heiland S, Luick R, Vedel D, Ammermann K, Wiersbinski N (Hrsg) Die Energiewende im Spannungsfeld energiepolitischer Ziele, gesellschaftlicher Akzeptanz und naturschutzfachlicher Anforderungen. Ergebnisse des gleichnamigen $\mathrm{F}+\mathrm{E}$ Vorhabens des Bundesamtes für Naturschutz. Bundesamt für Naturschutz, Bonn, S 4-17

Deutsche Windguard (2020) Status des Windenergieausbaus an Land in Deutschland. Jahr 2019. Deutsche WindGuard, Varel

Drews J (2018) Risikokommunikation und Krisenkommunikation. Kommunikation von Behörden und die Erwartungen von Journalisten. Springer, Wiesbaden

Dürrenberger G (2013) EMF-Risikokommunikation. Herausforderungen und Chancen für die Strombranche

Duve C, Eidenmüller H, Hacke A, Fries M (2011) Mediation in der Wirtschaft. Wege zum professionellen Konfliktmanagement. Otto Schmidt, Köln

Eltrop L, Härdtlein M, Jenssen T, Özdemir ED, Henßler M, Kruck C (2014) Dachleitfaden Bioenergie. Grundlagen und Planung von Bioenergieprojekten. Fachagentur Nachwachsende Rohstoffe e. V. (FNR), Gülzow

EnergieAgentur.NRW (2014) Windenergievorhaben und Akzeptanz. Bürgerbeteiligung am Planungsverfahren als integratives Projektmanagement. EnergieAgentur.NRW, Düsseldorf

Ermisch M, Hildebrandt I, Kaiser M, Packroff J, Wedell P, Zipf C (2018) Gemeinsam gewinnen - Windenergie vor Ort. Ein Grundlagenpapier zu den Themen Wertschöpfung, Bürgerbeteiligung und Akzeptanz. BWE, Berlin

Ewen C, Gabriel OW, Ziekow J (2013) Bürgerdialog bei der Infrastrukturplanung: Erwartungen und Wirklichkeit. Was man aus dem Runden Tisch Pumpspeicherwerk Atdorf lernen kann. Schriften zur Evaluationsforschung. Nomos, Baden-Baden

Fehrentz L (2020) Kommunikationsmanagement bei Bau- und Infrastrukturprojekten. Erfolgsfaktoren am Beispiel des ThyssenKrupp Testturms in Rottweil. In: Brettschneider F (Hrsg) Bau- und Infrastrukturprojekte. Dialogorientierte Kommunikation als Erfolgsfaktor. Springer, Wiesbaden, S 125-224

Feindt PH, Saretzki T (Hrsg) (2010) Umwelt- und Technikkonflikte. VS, Wiesbaden

Fischer R (2010) Konflikte um verrückte Kühe? Risiko- und Interessenskonflikte am Beispiel der europäischen BSE-Politik. In: Feindt PH, Saretzki T (Hrsg) Umwelt- und Technikkonflikte. VS, Wiesbaden, S 123-142

Freimuth V, Linnan HW, Potter P (2000) Communicating the threat of emerging infections to the public. Emerg Infect Dis 6(4):337-347

Fröhlich R, Szyszka P, Bentele G (Hrsg) (2015) Handbuch der Public Relations. Wissenschaftliche Grundlagen und berufliches Handeln. Mit Lexikon. Springer, Wiesbaden

Fuhrberg R, Umansky D (2016) Good guys vs. bad guys? Konflikte zwischen Selbst- und Fremdbild der Akteure als kommunikative Herausforderung für die Bürgerbeteiligung beim Übertragungsnetzausbau. In: Wissenschaftsdialog 2016. Tagungsband. Bundes- netzagentur für Elektrizität, Gas, Telekommunikation, Post und Eisenbahnen, Bonn, S 116-129

Geis A (2010) Beteiligungsverfahren zwischen Politikberatung und Konfliktregelung: Die Frankfurter Flughafen-Mediation. In: Feindt PH, Saretzki T (Hrsg) Umwelt- und Technikkonflikte. VS, Wiesbaden, S 259-274

Glasl F (2011) Konfliktmanagement. In: Meyer B (Hrsg) Konfliktregelung und Friedensstrategien. VS, Wiesbaden, S 125-145

Grünwald R, Ahmels P, Banthien H, Bimesdörfer K, Grünert J, Revermann C (2015) Handlungsmöglichkeiten für Kommunikation und Beteiligung beim Stromnetzausbau. Ein Praxishandbuch für Abgeordnete. TAB-Hintergrundpapier, Bd. 20. Büro für Technikfolgen-Abschätzung (TAB), Berlin

Hampel J, Torgersen H (2010) Der Konflikt um die Grüne Gentechnik und seine regulative Rahmung. Frames, Gates und die Veränderung der europäischen Politik zur Grünen Gentechnik. In: Feindt PH, Saretzki T (Hrsg) Umwelt- und Technikkonflikte. VS, Wiesbaden, S 143-162

Hänlein R, El Alaoui A (2015) Beteiligung und Transparenz bei der Stromnetzplanung. Handlungsempfehlungen vom BESTGRIDProjekt. Handbuch - Teil 1. Germanwatch e. V., Berlin

Heath RL, O'Hair D (Hrsg) (2009) Handbook of risk and crisis communication. Routledge, New York

Heger I (2016) Konfliktlandschaften der Energiewende - Bürgerproteste im Fokus. Kommunikative Herausforderungen in Energiekonflikten. In: Demuth B, Heiland S, Luick R, Vedel D, Ammermann K, Wiersbinski N (Hrsg) Die Energiewende im Spannungsfeld energiepolitischer Ziele, gesellschaftlicher Akzeptanz und naturschutzfachlicher Anforderungen. Ergebnisse des gleichnamigen $\mathrm{F}+\mathrm{E}$ Vorhabens des Bundesamtes für Naturschutz. Bundesamt für Naturschutz, Bonn, S 46-52

Hellmuth N, Jakobs E-M (2019a) Partizipationsformate für Stromnetzausbauprojekte im ruralen Raum. In: Fraune C, Knodt M, Gölz S, Langer K (Hrsg) Akzeptanz und politische Partizipation in der Energietransformation: Gesellschaftliche Herausforderungen jenseits von Technik und Ressourcenausstattung. Springer, Wiesbaden, S 183-207

Hellmuth N, Jakobs E-M (2019b) The unknown stakeholder. Energy communication for farmers. In: Proceedings of the IEEE International Professional Communication Conference (ProComm), 23.-26.07.2019, Aachen (DEU). IEEE, Piscataway, S 1-7

Hellmuth N, Jakobs E-M (2020) Informiertheit und Datenschutz beim Smart Metering. Z Energiewirtsch 44(1):15-29

Hoeft C, Messinger-Zimmer S, Zilles J (2017) Einleitung. In: Hoeft C, Messinger-Zimmer S, Zilles J (Hrsg) Bürgerproteste in Zeiten der Energiewende. Lokale Konflikte um Windkraft, Stromtrassen und Fracking. transcript, Bielefeld, S 9-42

Holstenkamp L, Radtke J (Hrsg) (2018) Handbuch Energiewende und Partizipation. Springer VS, Wiesbaden

Hübner G, Pohl J (2014) Akzeptanz der Offshore-Windenergienutzung. Abschlussbericht. Martin-Luther-Universität Halle-Wittenberg, Halle

Huge A, Roßnagel A (2018) Möglichkeiten der Öffentlichkeitsbeteiligung in Planungs- und genehmigungsverfahren von Windenergieanlagen. In: Holstenkamp L, Radtke J (Hrsg) Handbuch Energiewende und Partizipation. Springer VS, Wiesbaden, S 613-625

Jakobs E-M (2019) Technikakzeptanz und -kommunikation - Ein vielschichtiges Konstrukt. In: Fraune C, Knodt M, Gölz S, Langer K (Hrsg) Akzeptanz und politische Partizipation in der Energietransformation: Gesellschaftliche Herausforderungen jenseits von Technik und Ressourcenausstattung. Springer, Wiesbaden, S 301-321

Jobst C (2010) Ein lokaler Umweltkonflikt in Latenz: grüne Gentechnik und Entwicklungspfade der Pflanzenbiotechnologie. In: Feindt PH, Saretzki T (Hrsg) Umwelt- und Technikkonflikte. VS, Wiesbaden, S 163-180 
Kepplinger HM (2015) Konflikt- und Krisenkommunikation. In: Fröhlich R, Szyszka P, Bentele G (Hrsg) Handbuch der Public Relations. Wissenschaftliche Grundlagen und berufliches Handeln. Mit Lexikon. Springer, Wiesbaden, S 993-1000

Kluge J (2018) Protestler als Zielgruppe für Kommunikation bei Energie- und Infrastrukturprojekten. Apprimus-Verlag, Aachen

Krause D (1996) Luhmann-Lexikon: eine Einführung in das Gesamtwerk von Niklas Luhmann. Enke, Stuttgart

Krebber F (2016) Kommunikation bei Infrastrukturprojekten. In: Krebber F (Hrsg) Akzeptanz durch inputorientierte Organisationskommunikation. Infrastrukturprojekte und der Wandel der Unternehmenskommunikation. Springer, Wiesbaden, S 106-137

Kriegel K (2011) Verlustreiche Konfliktbearbeitung in deutschen Unternehmen. Mediation als gewinnbringende Alternative? Intercult J 9(11):3-25

Mast C, Stehle H (2016) Energieprojekte im öffentlichen Diskurs. Erwartungen und Themeninteressen der Bevölkerung. Springer, Wiesbaden

Mayring P (2010) Qualitative Inhaltsanalyse. Grundlagen und Techniken Bd. 11. Beltz, Weinheim

Möller C, Zdiara K (2017) Öffentlichkeitsbeteiligung beim Netzausbau. Mit informeller Beteiligung das formelle Verfahren vorbereiten und begleiten. eNewsletter Netzwerk Bürgerbeteiligung, Bd. 03/2017. Arcadis Germany, Darmstadt

Müller CE (2020) Why do residents participate in high-voltage transmission line planning procedures? Findings from two power grid expansion regions in Germany. German Research Institute for Public Administration, Speyer ((Preprint))

Nanz P, Fritsche M (2012) Handbuch Bürgerbeteiligung. Verfahren und Akteure, Chancen und Grenzen. Schriftenreihe BpB, Bd. 1200. bpb, Bonn

Naumann M, Becker S, Moss T (2017) Energiekonflikte: zuerst verstehen, dann nutzen. Ökol Wirtsch 32(3):30-34. https://doi.org/10. 14512/OEW32033

Ohlhorst D, Schön S (2010) Windenergienutzung in Deutschland im dynamischen Wandel von Konfliktkonstellationen und Konflikttypen. In: Feindt PH, Saretzki T (Hrsg) Umwelt- und Technikkonflikte. VS, Wiesbaden, S 198-218

Oppermann B, Renn O (2019) Partizipation und Kommunikation in der Energiewende (Schriftenreihe Energiesysteme der Zukunft). acatech, München

Park C-K, Kim H-J, Kim Y-S (2014) A study of factors enhancing smart grid consumer engagement. Energy Policy 72:211-218

Reimer E, Jakobs E-M, Borg A, Trevisan B (2015) New ways to develop professional communication concepts. In: Proceedings of the IEEE International Communication Conference (ProComm), 12.-15.07.2015, Limerick (IRL). IEEE, Piscataway, S 97-103

Renn O (2011) Wissen und Moral - Stadien der Risikowahrnehmung. In: Ende des Atomzeitalters? APuZ, Bd. 46-47, S 3-7 (61. Jahrgang)

Renn O (2015) Aspekte der Energiewende aus sozialwissenschaftlicher Perspektive. Analyse aus der Schriftenreihe Energiesysteme der Zukunft. acatech, München

Reynolds B, Seeger M (2005) Crisis and emergency risk communication as an integrative model. J Health Commun 10(1):43-55

Riede M (2013) Determinanten erfolgreicher Stakeholderdialoge. Erfolgsfaktoren von Dialogverfahren zwischen Unternehmen und Nicht-Regierungsorganisationen. uwf 21(1-2):45-50

Roßnagel A, Ewen C, Götz K, Hefter T, Hentschel A, Huge A, Schönfeder C (2014) Mit Interessengegensätzen fair umgehen - zum Einbezug der Öffentlichkeit in Entscheidungsprozesse zu dezentralen Energieanlagen. ZNER 4:329-337

Rowley J, Slack F (2004) Conducting a Literature Review. Manag Res News 27(6):31-39

Ruhrmann G (2015) Risiko und Risikokommunikation. In: Fröhlich R, Szyszka P, Bentele G (Hrsg) Handbuch der Public Relations. Wissenschaftliche Grundlagen und berufliches Handeln. Mit Lexikon. Springer, Wiesbaden, S 977-992
RWE AG (2012) Akzeptanz für Großprojekte. Eine Standortbestimmung über Chancen und Grenzen der Bürgerbeteiligung in Deutschland

Saretzki T (2010) Umwelt- und Technikkonflikte: Theorien, Fragestellungen, Forschungsperspektiven. In: Feindt PH, Saretzki T (Hrsg) Umwelt- und Technikkonflikte. VS, Wiesbaden, S 33-53

Scheffran J (2010) Energiekonflikte, Klimawandel und nachhaltige Entwicklung. In: Imbusch P, Zoll R (Hrsg) Friedens- und Konfliktforschung. Eine Einführung. VS, Wiesbaden, S 333-352

Schmalz IM (2019) Akzeptanz von Großprojekten. Eine Betrachtung von Konflikten, Kosten- und Nutzenaspekten und Kommunikation. VS, Wiesbaden

Schönrock E (2016) CSR-Kommunikation 3.0: Basis für eine erfolgreiche Energiewende, Bürgerbeteiligung und Akzeptanz von Großprojekten. In: Hildebrandt A, Landhäußer W (Hrsg) CSR und Energiewirtschaft. Gabler, Heidelberg, S 405-415

Schulz J (2001) Management von Risiko- und Krisenkommunikation - zur Bestandserhaltung und Anschlussfähigkeit von Kommunikationssystemen. Dissertation, Humboldt-Universität, Berlin

Schwarz A (2015) Strategische Krisenkommunikation von Organisationen. In: Fröhlich R, Szyszka P, Bentele G (Hrsg) Handbuch der Public Relations. Wissenschaftliche Grundlagen und berufliches Handeln. Mit Lexikon. Springer, Wiesbaden, S 1001-1016

Schwarz G (1990) Konflikt-Management. Gabler, Wiesbaden

Schweizer-Ries P, Rau I, Zoellner J (2010) Projektabschlussbericht der Forschungsgruppe Umweltpsychologie: Aktivität und Teilhabe Akzeptanz Erneuerbarer Energien durch Beteiligung steigern

TechnikRadar (2018) Was die Deutschen über Technik denken. Acatech - Deutsche Akademie für Technikwissenschaften und Körber-Stiftung, München, Hamburg

Verein Deutscher Ingenieure e. V. (VDI) (2014) VDI 7001. Frühe Öffentlichkeitsbeteiligung bei Industrie- und Infrastrukturprojekten. Beuth, Berlin

Verein Deutscher Ingenieure e. V. (VDI) (2015) VDI 7000. Kommunikation und Öffentlichkeitsbeteiligung bei Planung und Bau von Infrastrukturprojekten. Standards für die Leistungsphasen der Ingenieure. Beut, Berlin

Wachholz C (2008) NABU. Kommunikationsratgeber zum Ausbau Erneuerbarer Energien. NABU, Berlin

Weber D, Brian M (2014) Öffentlichkeitsarbeit für Geothermieprojekte. Planung, strategische Ausrichtung und Umsetzung von Kommunikationsmaßnahmen durch Projektbetreiber. Leitfaden, Bd. 3. Technische Informationsbibliothek, Universitätsbibliothek Freiburg, Freiburg (für Enerchange - Agentur für erneuerbare Energien)

Van Wee B, Banister D (2015) How to write a literature review paper? Transport Rev 36(2):278-288

Weitze M-D, Renn O (2019) Technikkommunikation, Risikobewertung und Risikokommunikation. In: Hennecke M, Skrotzki B (Hrsg) HÜTTE - Das Ingenieurwissen. Springer, Berlin, Heidelberg, New York

Wiedemann PM, Carius R, Henschel C, Kastenholz H, Nothdurft W, Ruff F, Uth HJ (2000) Risikokommunikation für Unternehmen. VDI-Verlag, Düsseldorf

Wolf I, Stadler T, Pinto DL (2020) Soziales Nachhaltigkeitsbarometer der Energiewende 2019. IASS, Potsdam

Wunderlich C (2012) Akzeptanz und Bürgerbeteiligung für Erneuerbare Energien. Erkenntnisse aus Akzeptanz- und Partizipationsforschung. Renews, Bd. 60. AEE, Berlin

Ziekow J, Barth R, Schütte S, Ewen C (2014a) Konfliktdialog bei der Zulassung von Vorhaben der Energiewende. Leitfaden für Behörden. Grundsätze informeller Konfliktdialoge

Ziekow J, Barth R, Schütte S, Ewen C (2014b) Konfliktdialog bei der Zulassung von Vorhaben der Energiewende. Leitfaden für Behörden. Konfliktdialog bei Biomasseanlagen

Ziekow J, Barth R, Schütte S, Ewen C (2014c) Konfliktdialog bei der Zulassung von Vorhaben der Energiewende. Leitfaden für Behörden. Konfliktdialog bei Höchstspannungsanlagen 
Ziekow J, Barth R, Schütte S, Ewen C (2014d) Konfliktdialog bei der Zulassung von Vorhaben der Energiewende. Leitfaden für Behörden. Konfliktdialog bei Pumpspeicherkraftwerken

Ziekow J, Barth R, Schütte S, Ewen C (2014e) Konfliktdialog bei der Zulassung von Vorhaben der Energiewende. Leitfaden für Behörden. Konfliktdialog bei tiefer Geothermie
Ziekow J, Barth R, Schütte S, Ewen C (2014f) Konfliktdialog bei der Zulassung von Vorhaben der Energiewende. Leitfaden für Behörden. Konfliktdialog bei Windenergieanlagen 